\title{
BMJ Open Are prolonged sitting and sleep restriction a dual curse for the modern workforce? a randomised controlled trial protocol
}

\author{
Grace E Vincent (D) , ${ }^{1}$ Charlotte C Gupta (D) , ${ }^{1}$ Madeline Sprajcer (D) , 1 \\ Corneel Vandelanotte (1D, , Mitch J Duncan (1) , 3,4 Phil Tucker (D) ,5,6 \\ Michele Lastella (D) , ${ }^{1}$ Georgia A Tuckwell (D) , ${ }^{1}$ Sally A Ferguson (iD ${ }^{1}$
}

To cite: Vincent GE, Gupta CC, Sprajcer M, et al. Are prolonged sitting and sleep restriction a dual curse for the modern workforce? a randomised controlled trial protocol. BMJ Open 2020;10:e040613. doi:10.1136/ bmjopen-2020-040613

- Prepublication history and additional material for this paper are available online. To view these files, please visit the journal online (http://dx.doi. org/10.1136/bmjopen-2020040613).

Received 19 May 2020

Revised 27 May 2020

Accepted 28 May 2020
Check for updates

(C) Author(s) (or their employer(s)) 2020. Re-use permitted under CC BY-NC. No commercial re-use. See rights and permissions. Published by BMJ.

For numbered affiliations see end of article.

Correspondence to

Dr Grace E Vincent;

g.vincent@cqu.edu.au

\section{ABSTRACT}

Introduction Prolonged sitting and inadequate sleep are a growing concern in society and are associated with impairments to cardiometabolic health and cognitive performance. However, the combined effect of prolonged sitting and inadequate sleep on measures of health and cognitive performance are unknown. In addition, the circadian disruption caused by shiftwork may further impact workers' cardiometabolic health and cognitive performance. This protocol paper outlines the methodology for exploring the impact of simultaneous exposure to prolonged sitting, sleep restriction and circadian disruption on cardiometabolic and cognitive performance outcomes.

Methods and analysis This between-subjects study will recruit 208 males and females to complete a 7-day in-laboratory experimental protocol (1 Adaptation Day, 5 Experimental Days and 1 Recovery Day). Participants will be allocated to one of eight conditions that include all possible combinations of the following: dayshift or nightshift, sitting or breaking up sitting and 5 hour or 9 hour sleep opportunity. On arrival to the laboratory, participants will be provided with a 9 hour baseline sleep opportunity (22:00 to 07:00) and complete five simulated work shifts (09:00 to 17:30 in the dayshift condition and 22:00 to $06: 30$ in the nightshift condition) followed by a 9 hour recovery sleep opportunity (22:00 to 07:00). During the work shifts participants in the sitting condition will remain seated, while participants in the breaking up sitting condition will complete 3-min bouts of light-intensity walking every 30 mins on a motorised treadmill. Sleep opportunities will be 9 hour or 5 hour. Primary outcome measures include continuously measured interstitial blood glucose, heart rate and blood pressure, and a cognitive performance and self-perceived capacity testing battery completed five times per shift. Analyses will be conducted using linear mixed models.

Ethics and dissemination The CQUniversity Human Ethics Committee has approved this study (0000021914). All participants who have already completed the protocol have provided informed consent. Study findings will be disseminated via scientific publications and conference presentations.

Trial registration details This study has been registered on Australian New Zealand Clinical Trials Registry

\section{Strengths and limitations of this study}

- This is the first study to investigate the combined effect of prolonged sitting and sleep restriction during dayshifts and nightshifts on cardiometabolic and cognitive performance outcomes.

- A strength of the study is the highly controlled, rigorous experimental in-laboratory protocol.

- This research will contribute to the evidence-base for workplace and public health guidelines and there will be an opportunity to present targeted advice to workers about sleep and prolonged sitting.

- A limitation is the (apparently) healthy, nonshiftworking participants who may not be representative of shiftworking or other working adults.

- The long-term impacts of prolonged sitting and sleep restriction on health cannot be elucidated from this study.

(12619001516178) and is currently in the pre-results stage.

\section{INTRODUCTION}

The majority of employed adults in Western countries $(80 \%)$ spend time sitting for work, ${ }^{1}$ with 10 to 16 hours of sedentary behaviour per week reported in Australia ${ }^{1}$ and in the USA. ${ }^{2}$ This is problematic, as prolonged sitting contributes to the health and economic burden of physical inactivity. In an Australian context, the healthcare and lost productivity costs associated with physical inactivity are in excess of $\$ \mathrm{~A} 1.6$ billion (Australian Dollar) annually. ${ }^{3}$ Further, $45 \%$ of Australians do not obtain the recommended 7 to 9 hours of sleep. ${ }^{4}$ The economic effect of sleep restriction in Australia totals an estimated \$A66 billion, including \$A18billion in lost productivity. ${ }^{5}$ While the issues associated with sleep restriction and physical inactivity are part of distinct public health interventions 
and educational campaigns, ${ }^{6}$ to date, no study has investigated the impacts of prolonged sitting and sleep restriction in combination.

Both prolonged sitting and sleep restriction are associated with numerous long-term health effects, such as cardiometabolic disease including type 2 diabetes and cardiovascular disease. ${ }^{7-9}$ In addition, both prolonged sitting and sleep restriction are associated with an increased risk of mortality, ${ }^{10}{ }^{11}$ with a dose-response relationship existing between prolonged sitting and mortality. ${ }^{12}$ The associations between higher levels of sedentary behaviour, cardiovascular disease and all-cause mortality are modified by physical activity, with higher risk observed among populations engaging in the lowest amounts of physical activity. ${ }^{13-15}$ To improve cardiometabolic health markers and mortality, research has investigated the effect of lightintensity physical activity. ${ }^{13}$ For example, breaking up sitting with 2 to $3 \mathrm{~min}$ of light-intensity physical activity every 20 to $30 \mathrm{~min}$ results in decreased resting blood pressure and reduced rates of all-cause mortality. ${ }^{16-19}$ This differs depending on task duration, with short bouts of physical activity shown to be more effective than a single, continuous bout $(30 \mathrm{~min}) \cdot{ }^{20}$ Of note, these studies have not controlled for, nor reported, prior sleep duration. ${ }^{21}$ This is a gap in the current literature as sleep restriction is also associated with negative changes to the same cardiometabolic markers that are affected by prolonged sitting. ${ }^{22} 23$ Pilot work from our group found that breaking up sitting did not improve these cardiometabolic outcomes in sleep restricted (5 hour sleep opportunity) individuals when compared with well-rested individuals (9 hour sleep opportunity). ${ }^{20}{ }^{24}$ Therefore, breaking up sitting may not be as effective in sleep restricted individuals compared with well-rested individuals. ${ }^{25} 26$ This suggests that, sleep restricted individuals may not benefit from current recommendations to break up sitting and a tailored set of recommendations may be required.

Cognitive performance is affected by both prolonged sitting and sleep restriction. Sleep restriction leads to impairments in many cognitive components of work performance, including sustained attention and working memory, as well as increased self-reported fatigue. ${ }^{26-28}$ Further, there is an increase in the likelihood of fatiguerelated driving accidents. ${ }^{29}$ Similarly, impaired alertness due to increased sleepiness is worse in conditions of prolonged sitting, when compared with breaking up sitting with brief bouts of physical activity. ${ }^{20}$ Overall work productivity was also shown to be lower under conditions of prolonged sitting compared with a breaking up sitting condition. ${ }^{30}$ However, to date no studies have investigated the effect of breaking up sitting when sleep restricted on measures of cognitive performance.

The gap in evidence regarding the impacts of prolonged sitting and sleep restriction is particularly relevant to the $20 \%$ of the worldwide workforce who undertake shiftwork, ${ }^{31}$ including 1.9 million workers in Australia. ${ }^{32}$ Shiftwork may include early morning shifts, evening shifts and nightwork ${ }^{33}$ and is the reality for an increasing proportion of the workforce worldwide. ${ }^{34}$ Prolonged sitting and sleep restriction are more common in shiftworkers than day workers, ${ }^{25} 35$ and this is especially concerning for those who are working during the night who also experience disruption to their biological sleep/wake cycle (circadian disruption). ${ }^{33}$ This may partly explain the higher rates of cardiometabolic disease in shiftworkers compared with dayworkers. ${ }^{33}$ While in-shift breaks mitigate against both cognitive performance impairment and reduced alertness on shift, ${ }^{36}{ }^{37}$ it is unknown which aspect of the break (eg, cognitive rest, change of task, movement) contributes to improved work performance. This knowledge is important to provide targeted recommendations for in-shift behaviour. To mitigate the health risks of shiftwork, it is important to understand the combined effects of prolonged sitting and sleep restriction when working at night, as well as during the day.

The aim of the present study is to investigate the impact of simultaneous exposure to prolonged sitting, sleep restriction and circadian disruption, as well as the effects of breaking up sitting on these relationships. Therefore, this project will contribute foundational knowledge on the cardiometabolic and cognitive performance impact of exposure to prolonged sitting, sleep restriction and circadian disruption. The effectiveness of breaking up sitting during sleep restriction and circadian disruption on cognitive performance will also be tested. Positive outcomes for cardiometabolic and cognitive performance will benefit worker's health and well-being, increase productivity and organisational outcomes, as well as potentially reducing the likelihood of fatigue-related accidents and injuries.

\section{METHODS AND ANALYSIS}

\section{Study design}

This proposed study will use a between-subjects factorial design $(2 \times 2 \times 2$; conditions described below). Participants will live in the Appleton Institute Sleep Laboratory at Central Queensland University Adelaide Campus for 7 days (1 Adaptation Day, 5 Experimental Days and 1 Recovery Day). This study has been approved by the Central Queensland University Human Research Ethics Committee (0000021914) and registered with the Australian New Zealand Clinical Trials Registry. Table 1 provides a summary of the five overarching research questions and hypotheses for this project.

\section{Conditions}

Participants will be allocated to one of eight conditions (table 2): 2 (dayshift or nightshift) $\times 2$ (sitting or breaking up sitting $) \times 2$ ( 5 hour or 9 hour sleep opportunity). The sleep laboratory has capacity for six participants to participate in the study at one time (known as a study 'run'). Participants will be allocated at the run level to a dayshift or nightshift condition, and a 5 hour or 9 hour sleep opportunity condition (see figure 1). Within a run of 6 participants, three participants will be randomised to the sitting condition and three will be randomised to the 
Table 1 Major research questions and related hypotheses

\begin{tabular}{ll} 
Research question & Hypothesis \\
\hline $\begin{array}{l}\text { What is the cardiometabolic impact of simultaneous } \\
\text { exposure to prolonged sitting and sleep restriction? }\end{array}$ & $\begin{array}{l}\text { Simultaneous exposure to prolonged sitting and sleep restriction } \\
\text { will result in poorer cardiometabolic outcomes, compared with } \\
\text { prolonged sitting or sleep restriction alone. }\end{array}$ \\
$\begin{array}{ll}\text { What is the cardiometabolic impact of breaking up sitting } \\
\text { in sleep restricted and non-sleep restricted individuals? }\end{array}$ & $\begin{array}{l}\text { Breaking up sitting will benefit cardiometabolic outcomes in non- } \\
\text { sleep restricted individuals, but not in sleep restricted individuals. }\end{array}$ \\
$\begin{array}{l}\text { What is the cardiometabolic impact of breaking up sitting } \\
\text { during the day and night? }\end{array}$ & $\begin{array}{l}\text { Breaking up sitting will result in poorer cardiometabolic outcomes at } \\
\text { night, compared with the day. }\end{array}$ \\
$\begin{array}{ll}\text { What is the cognitive performance impact of breaking } \\
\text { up sitting in sleep restricted and non-sleep restricted } \\
\text { individuals? }\end{array}$ & $\begin{array}{l}\text { Breaking up sitting will benefit cognitive performance in non-sleep } \\
\text { restricted individuals, but not sleep restricted individuals. }\end{array}$ \\
$\begin{array}{ll}\text { What is the cognitive performance impact of breaking up } \\
\text { sitting during the day and night? }\end{array}$ & $\begin{array}{l}\text { Breaking up sitting will benefit cognitive performance during the day, } \\
\text { but not at night. }\end{array}$
\end{tabular}

breaking up sitting condition. The randomisation process will stratify participants by gender (male or female) and body mass index (BMI; 18 to $29 \mathrm{~kg} / \mathrm{m}^{2}$ ) to ensure equal distribution across conditions. Randomisation will be done by the researchers who are conducting the laboratory protocol and the researchers will not be blinded to study condition.

\section{Participant recruitment and screening procedure}

A total of 208 participants (104 male:104 female) will be recruited with 26 participants in each of the eight conditions. To ensure adequate participant enrolment, participants will be recruited via online advertisements, flyers and word of mouth from the Adelaide (Australia) region. Participant recruitment commenced in November 2019 and is ongoing. There has been a break in recruitment due to COVID-19 and this break will last until government restrictions permit continuance. Participants will provide informed consent (see online supplementary file 1) and will be compensated for their time $(\$ A 780)$. Participants will be screened prior to participation using a series of standard questionnaires to ensure that participants meet the inclusion and exclusion criteria (table 3). These criteria are consistent with standard screening procedures at the Appleton Institute Sleep Laboratory ${ }^{38-40}$ and other sleep laboratories. ${ }^{4142}$ Eligible participants will then visit the laboratory for a physical screening session. If participants are still eligible, pre-study screening procedures will be completed 1 week prior to study commencement. See figure 1 for an overview of the screening procedure.

\section{Initial online questionnaire screening}

Initial participant eligibility will be determined by the completion of an online questionnaire through Qualtrics (Provo, Utah, USA), assessing the criteria listed in table 3.

\section{Pittsburgh Sleep Quality Index}

Participants will complete the Pittsburgh Sleep Quality Index (PSQI), which provides a score that is reflective of overall typical sleep quality. ${ }^{43}$ This scale consists of 19 items which assess sleep quality and duration over the previous month. These items assess seven separate components, including subjective sleep quality, sleep onset latency, sleep duration, sleep efficiency, sleep disturbances, use of sleep medication and daytime dysfunction. Each question requires an answer resulting in a score of 0 to 3 which are then summed to create an overall score that can range from 0 to 21, with higher scores indicating poorer sleep quality. ${ }^{43}$ Individuals will be excluded from participation if they have a PSQI score greater than 5, which is the clinical standard for poor sleep quality. ${ }^{43}$ This measure has been extensively validated and assessed for reliability, producing a Cronbach $\alpha=0.83$ with both clinical $^{43} 45$ and non-clinical populations. ${ }^{46}$

\section{Epworth Sleepiness Scale}

Participants will complete the Epworth Sleepiness Scale (ESS). ${ }^{47}$ This 8-item questionnaire measures typical daytime sleepiness, with higher scores representative of higher sleepiness. This scale consists of eight items describing times where an individual may fall asleep, such as 'sitting quietly after lunch without alcohol' and 'in a car, while stopped for a few minutes in traffic'. Participants are asked to rate how likely it is that the participant would 'doze off' during these situations. Ratings go from 0 ('would never doze') to 3 ('high chance of dozing'). Potential scores range from 0 to 24 (0 to 10 indicating no/ low daytime sleepiness, 11 to 14 indicating mild daytime sleepiness, 15 to 17 indicating moderate daytime sleepiness and $>17$ indicating severe daytime sleepiness). ${ }^{47}$

Table 2 Overview of the eight study conditions

\section{Dayshift \\ Nightshift}

\begin{tabular}{|c|c|c|c|c|}
\hline Sitting & 9 hour sleep opportunity & 5 hour sleep opportunity & 9 hour sleep opportunity & 5 hour sleep opportunity \\
\hline Breaking up sitting & 9 hour sleep opportunity & 5 hour sleep opportunity & 9 hour sleep opportunity & 5 hour sleep opportunity \\
\hline
\end{tabular}




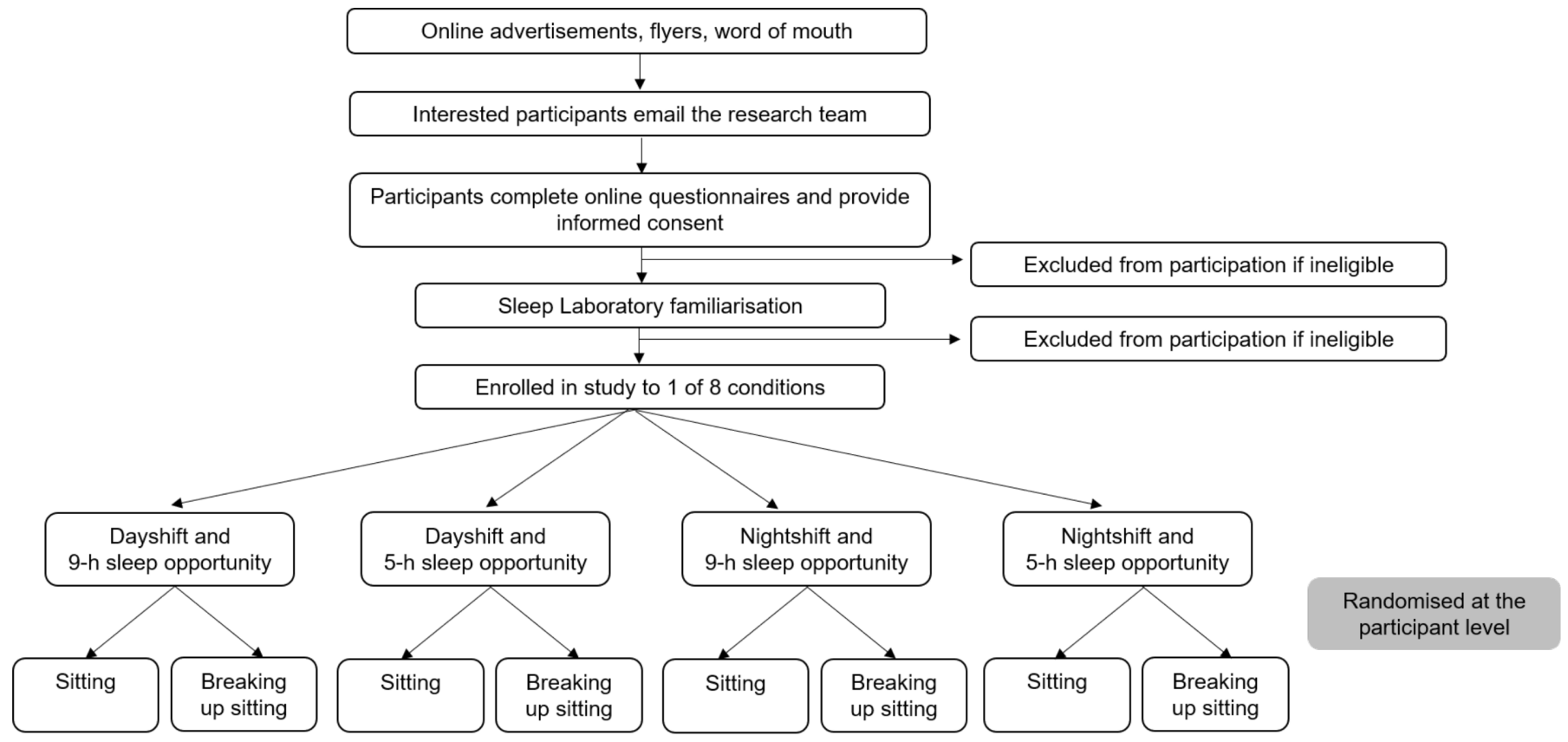

Figure 1 Flowchart of the screening process and allocation to study condition.

Participants with an ESS score higher than or equal to 10 will be excluded from the study. ${ }^{47}$

\section{Morningness-Eveningness Questionnaire}

The Morningness-Eveningness Questionnaire (MEQ) will be used to screen participants based on chronotype (ie, whether the participant is a 'morning person' or an 'evening person'). The $\mathrm{MEQ}^{48}$ is a 19-item questionnaire asking questions such as 'if you got into bed at 11:00 PM, how tired would you be?', and 'at what time of day do you feel you become tired as a result of need for sleep?'. Scores between 0 to 6 are allocated for each response resulting in a total maximum score of 86 . Scores categorise respondents into the following categories: definitely morning type (70 to 86 ), moderately morning type (59 to 69 ), neither type (42 to 58), moderately evening type (31 to 41 ) and definitely evening type (16-30). ${ }^{48}$ Participants will be excluded from the study if the participants are categorised as either 'definitely evening types' or 'definitely morning types', as these categories represent extreme chronotypes.

\section{Berlin Questionnaire}

The Berlin Questionnaire will be used to screen for obstructive sleep apnoea. ${ }^{49}$ This 10 -item questionnaire focusses on snoring and breathing irregularities during sleep, in addition to daytime dysfunction (ie, daytime fatigue). Questionnaire items are broken into three categories, with scores assigned point values and summed for each category. Category scores are determined to be either 'positive' or 'negative'. A positive score in two or more categories is indicative of a high-risk of obstructive sleep apnoea in the respondent. Participants who have two or three positive categories (high-risk) are to be excluded from participation in the current study.
Insomnia Severity Index

Participants will complete the Insomnia Severity Index to screen for clinically significant insomnia. ${ }^{50}$ This index consists of seven questions with scores ranging from 0 to 4 allocated based on each response. Scores from 0 to 7 are representative of 'absence of clinically significant insomnia'. Scores from 8 to 14 indicate subthreshold insomnia, with scores 15 and over representing either moderate (15 to 21 ) or severe insomnia (22 to 28 ). For this study, participants will be excluded if their Insomnia Severity Index score is $\geq 15$.

\section{Depression Anxiety Stress Scale}

The Depression Anxiety Stress Scale will be used to screen participants for severity of depression, anxiety and stress symptoms. ${ }^{51}$ This scale includes 21 items targeted towards identifying levels of depression, anxiety and stress, such as 'I found myself getting upset by quite trivial things' and 'I couldn't seem to experience any positive feeling at all'. Participants are asked to rate how much each statement applied to them over the past week. Statements were scored from 0 ('did not apply to me at all') to 3 ('applied to me very much or most of the time'). Scores are summed for each subscale (depression, anxiety and stress) and used to determine the severity of relevant symptoms. Severity ranged from 'normal' to 'very severe'. Please see table 4 for the score classification. Participants will be excluded if they have scores classified as 'severe' or 'very severe' for depression, anxiety or stress.

International Physical Activity Questionnaire

The International Physical Activity Questionnaire short form (IPAQ-SF) will be used to assess physical activity levels. ${ }^{52}$ The IPAQ-SF includes seven items that assess the frequency and duration of vigorous-intensity physical 
Table 3 Overview of inclusion and exclusion criteria

\begin{tabular}{|c|c|c|c|}
\hline Criterion & Scale & Inclusion criteria & Exclusion criteria \\
\hline Sleep quality & Pittsburgh Sleep Quality Index ${ }^{43}$ & Score $\leq 5$ & Score $>5$ \\
\hline Daytime sleepiness & Epworth Sleepiness Scale ${ }^{47}$ & Score $\leq 10$ & Score $>10$ \\
\hline Sleep apnoea & Berlin Questionnaire ${ }^{49}$ & 'Positive' scores in zero or one category & 'Positive' scores in two or three categories \\
\hline Insomnia & Insomnia Severity Index ${ }^{50}$ & Score $<15$ & Score $\geq 15$ \\
\hline Physical activity & $\begin{array}{l}\text { International Physical Activity } \\
\text { Questionnaire Short-Form }{ }^{166}\end{array}$ & 'low' scores & 'moderate' or 'high' scores \\
\hline Sedentary time & Workforce Sitting Questionnaire ${ }^{54}$ & $\geq 6$ hour sitting time/day & $<6$ hour sitting time/day \\
\hline Diet & Food Frequency Questionnaire ${ }^{56}$ & $\begin{array}{l}\text { Participants who are not vegan and } \\
\text { without significant food allergies }\end{array}$ & Vegan diet and significant food allergies \\
\hline $\begin{array}{l}\text { Menstrual phase (female } \\
\text { only) }\end{array}$ & Questions on menstrual phase $\mathrm{e}^{59}$ & $\begin{array}{l}\text { History of regular menstrual cycles and } \\
\text { no health concerns that impact menstrual } \\
\text { cycles }\end{array}$ & $\begin{array}{l}\text { Menopausal, history of irregular menstrual cycles } \\
\text { or current health concern that impacts menstrual } \\
\text { cycles }\end{array}$ \\
\hline Health concerns & General Health Questionnaire & No health concerns & $\begin{array}{l}\text { Serious health concerns that may impact } \\
\text { participant's ability to engage in physical activity, } \\
\text { sleep or cognitive ability. Previous or current } \\
\text { diagnosis of psychiatric and/or neurological } \\
\text { problems or sleep disorders. }\end{array}$ \\
\hline $\begin{array}{l}\text { Contraindications to } \\
\text { exercise }\end{array}$ & Adult Pre-Exercise Screening System ${ }^{58}$ & All 'no' responses to APSS & Any 'yes' responses to APSS \\
\hline Age & General Health Questionnaire & Age 18 to 35 & $<18$ or $>35$ years \\
\hline Weight & General Health Questionnaire & BMI 18 to $30 \mathrm{~kg} \cdot \mathrm{m}^{2}$ & $\mathrm{BMl}<18$ or $>30 \mathrm{~kg} \cdot \mathrm{m}^{2}$ \\
\hline $\begin{array}{l}\text { Typical bed and wake } \\
\text { times }\end{array}$ & General Health Questionnaire & $\begin{array}{l}\text { Habitual bedtimes } 22: 00 \text { to } 00: 00 \text { and } \\
\text { habitual wake times } 06: 00 \text { to } 08: 00 \text {. }\end{array}$ & $\begin{array}{l}\text { Habitual bedtimes outside of } 22: 00 \text { to } 00: 00 \text { or } \\
\text { habitual wake times outside of } 06: 00 \text { to } 08: 00 \text {. }\end{array}$ \\
\hline Napping & General Health Questionnaire & No history of habitual napping & History of habitual napping \\
\hline $\begin{array}{l}\text { Recent trans-meridian } \\
\text { travel }\end{array}$ & General Health Questionnaire & $\begin{array}{l}\text { No travel across multiple time zones in the } \\
\text { previous } 4 \text { weeks }\end{array}$ & $\begin{array}{l}\text { Travel across multiple time zones in the previous } \\
4 \text { weeks. }\end{array}$ \\
\hline Language & General Health Questionnaire & $\begin{array}{l}\text { Fluent in English (ie, able to communicate } \\
\text { and understand English and to be able to } \\
\text { complete questionnaires) }\end{array}$ & Not fluent in English \\
\hline Sleep disorder & General Health Questionnaire & No diagnosed sleep disorders & Any diagnosed sleep disorder \\
\hline
\end{tabular}

APSS, Adult Pre-Exercise Screening System; BMI, body mass index.

activity, moderate-intensity physical activity and walking. Standard scoring protocols will be used to determine the total metabolic equivalent of task (MET) minutes of physical activity using MET values of 3.3 for walking, 4.0 for moderate-intensity physical activity and 8.0 for vigorous-intensity physical activity, and subsequently classify participants' level of physical activity as either low, moderate or high. Participants in the present study will be excluded if their scores result in a classification of 'moderate' or 'high'.

Table 4 Classification of scores on the Depression Anxiety Scale

\begin{tabular}{llllll}
\hline & Normal & Mild & Moderate & Severe & Very severe \\
\hline Stress & $0-14$ & $15-18$ & $19-25$ & $26-33$ & $34+$ \\
Anxiety & $0-7$ & $8-9$ & $10-14$ & $15-19$ & $20+$ \\
Depression & $0-9$ & $10-13$ & $14-20$ & $21-27$ & $28+$ \\
\hline
\end{tabular}




\section{Workforce Sitting Questionnaire}

The Workforce Sitting Questionnaire (WSQ) will be used to assess sedentary behaviour. Sedentary behaviour is defined as waking behaviours that result in an energy expenditure of $\leq 1.5$ METs, while in a sitting, reclining or lying posture. ${ }^{53}$ The WSQ assesses the duration of sitting during transport, work, watching television, using a computer at home and other leisure activities on work and non-workdays. Standard scoring protocols will be used to estimate the average daily sitting time. ${ }^{54}$ This information is used to determine the average number of hours spent sitting on both work and non-workdays. Participants will be excluded from participation if they spend on average $<6$ hour sitting each day on work and non-workdays. ${ }^{55}$

\section{Food Frequency Questionnaire}

Participants will also complete the Food Frequency Questionnaire to determine their regular eating habits. This questionnaire has been validated and used previously in nutrition studies. ${ }^{5657}$ To complete this questionnaire, participants will provide estimated quantities of various food types. Food types are categorised as: (1) dairy food and alternative; (2) fruits; (3) vegetables, legumes and beans; (4) meat, fish, eggs and processed meat; (5) bread, cereal and starches; (6) beverages; (7) sweets, baked goods and snacks; (8) added salt, sugar and oil; (9) supplements and (10) others. Additional questions regarding the frequency with which participants consume fast food, meal types and dietary requirements are also included. Participants will also be excluded from the study if they are vegan or have significant food allergies or dietary requirements.

\section{Adult Pre-Exercise Screening System}

To ensure that participants do not have physical contraindications to physical exercise, the Adult Pre-Exercise Screening System will be completed. ${ }^{58}$ Participants will be excluded if they have a heart condition, previously had a stroke, experienced chest pains or dizziness during exercise, have had a serious asthma attack during the last 12 months or have diabetes.

\section{Menstrual phase - female only}

Menstrual phase will be determined by self-reported date of menses onset. This will be done by the forward counting method, where menstrual phase is determined by counting the number of days forward from the previous menses onset date. This method is standard in the literature, with a recent review indicating that $60 \%$ of relevant studies employed this method to determine menstrual phase. ${ }^{59}$ Participants will be asked to provide the start and end date, to the best of their knowledge, for as many previous cycles during the previous 12 months as they can remember or have record of. Participants will also be asked to estimate their average menstrual cycle length, ${ }^{60}$ in addition to providing information regarding menstrual cycle regularity and any health concerns that may impact menstrual cycles (eg, polycystic ovarian syndrome). Participants who have a history of irregular menstrual cycles (variation $>8$ days) or are pregnant will be excluded from the study. ${ }^{18}$

Participants who are currently using hormonal contraceptives will not be excluded from the study. Hormonal contraceptives result in the suppression of normal oestrogen and progestin cycles, replacing hormonal release with synthetic hormones. ${ }^{61}$ As a result, fewer hormonal fluctuations can be seen during periods where hormonal contraception is in use (ie, on days when individuals take an contraceptive pill or at all times when an intrauterine device is in use). ${ }^{61}$ However, variations in hormone production do occur at times when the oral contraceptive is not taken (ie, on days when the individual takes the sugar pill). As a result, female participants who were currently using hormonal contraceptives will be asked to complete the same menstruation tracking questionnaire as all other female participants, in addition to providing information regarding which hormonal contraceptive was currently in use (eg, oestrogen and progestin combination or progestin only).

\section{Physical screening}

If participants are eligible based on their completion of the online questionnaires, they will be invited to attend a familiarisation session at the sleep laboratory. Participants will be shown around the facility, complete a breaking up sitting familiarisation session, and given the opportunity to ask any questions regarding the study. Participant weight, height and waist circumference will be measured twice in the standing position and the means of the two measurements recorded.

\section{Height}

Participant height will be measured to the nearest $0.1 \mathrm{~cm}$ using a stadiometer. Participants will be asked to remove their shoes prior to measurement.

\section{Body weight}

Body weight will be measured via portable electronic calibrated scales (Salter, Model 9102, Thebarton, South Australia) and recorded to the nearest $0.1 \mathrm{~kg}$. Participants will be required to remove their jackets, shoes and any heavy items from their pockets prior to measurement.

\section{Body mass index}

Participant height and weight measurements (as opposed to self-reports) will be used to calculate their BMI (BMI=weight $(\mathrm{kg}) \times$ height $\left.\left(\mathrm{m}^{2}\right)\right)$. BMI is used to determine whether participants are within a healthy weight range. Based on guidelines provided by the WHO, healthy BMI scores range from 18.50 to 24.99 with overweight scores ranging from 25.00 to $29.99 .^{62}$

Previous studies have noted that $\sim 75 \%$ of shiftworkers have a BMI in the range of 18 to $30 \mathrm{kgm}^{2}{ }^{6364}$ Therefore, in the current study participants will be excluded if they have a BMI score of $\geq 30$. This exclusion criteria has also been used in other laboratory based shiftworking protocols. $^{6566}$ 


\section{Waist and hip circumference}

Waist and hip circumference will be measured during the laboratory familiarisation session. Using a standard flexible tape measure, measures will be taken from the umbilicus (waist circumference) and at the widest part of the hips (hip measurement). Based on the anthropometric measurements of weight $(\mathrm{kg})$, height $(\mathrm{cm})$, waist circumference $(\mathrm{cm})$ and hip circumference $(\mathrm{cm})$, the following parameters will be calculated: waist-to-hip ratio (WHR) and waist-to-height ratio (WHtR). For female participants the WHR cut-off is $<0.85$, as a WHR of $\geq 0.85$ is generally classified as being obese. For men, this cut-off point is $\geq 0.90 .{ }^{67}$ For both genders, a WHtR of $<0.50$ is required as a WHtR of $>0.50$ is typically representative of significantly increased health risks. ${ }^{68}$

\section{Breaking up sitting familiarisation}

All participants who attend the laboratory will also perform a breaking up sitting familiarisation session, which will consist of a 3 min treadmill walk at a lightintensity speed $(3.2 \mathrm{~km} / \mathrm{h}$ for all participants $)$ on a motorised treadmill (Healthrider H95T; Icon Health \& Fitness Inc, Utah, USA). All treadmills will be set to a $0 \%$ incline. Participants will then be asked to rate their perceived exertion on a standard Borg Rating of Perceived Exertion (RPE).$^{69}$ The Borg RPE scale ranges from 0 (no exertion at all) to 20 (maximal exertion). Additional exertion descriptors are provided for scores of 7.5 (extremely light), 9 (very light), 11 (light), 13 (somewhat hard), 15 (hard/heavy), 17 (very hard) and 19 (extremely hard). This familiarisation technique is in line with the previous research into the impact of breaking up sitting on glucose metabolism. ${ }^{70-72}$

\section{One-week prior to study commencement}

In the week prior to the study, participants will be required to follow a regular sleep schedule, with 9 hour time in bed each night. Participant bed times are required to be between 22:00 to 00:00 and rise times between 06:00 to 08:00. These bed and rise times were selected as they are 1 hour before and after the bed and rise times of the adaptation night.

Participants will also be asked to follow their normal diet during the week prior (ie, not make any sudden dietary changes). For the 48 hours prior to study commencement, participants will be instructed to refrain from moderateto-vigorous-intensity physical activity, to ensure no 'carry over' effects are seen during study participation. ${ }^{72}$ In addition, participants will be asked to not consume caffeine or alcohol during this 48 hours period. ${ }^{72}$

\section{Actigraphy and sleep diaries}

Participants will wear an activity monitor (ie, Actical MiniMitter/Respironics, Bend, Oregon) and a Whoop (CB Rank, Greater Boston, New England, USA) as objective measures of sleep prior to study commencement.

Activity monitors (Actical MiniMitter/Respironics, Bend, Oregon) are well validated as a measure of sleep time and wakefulness. ${ }^{73}$ The dimensions of this device are $2.8 \times 2.7 \times 1.0 \mathrm{~cm}$, and the weight is $17 \mathrm{~g} .{ }^{74}$ The monitor uses a piezoelectric omnidirectional accelerometer, sensitive to movements in all planes in the range of 0.5 to $3.0 \mathrm{~Hz}$, to measure the frequency, intensity and duration of human movement. ${ }^{75}$ Data will be analysed in $1 \mathrm{~min}$ epochs according to standard criteria, ${ }^{76} 77$ with raw data reported in units of counts. $\mathrm{min}^{-1}$. Data will be downloaded using Actical software (Phillips Actical MiniMitter, Respironics, Bend, Oregon, USA).

The Actical will be worn on their non-dominant wrist 24 hours per day except during water-based activities (eg, showering) and will complete a sleep diary for 1 week prior to the study. This will be done to corroborate selfreported bed and rise times and to ensure that participants do not have detectable sleep problems (eg, $<85 \%$ sleep efficiency). ${ }^{73}$ Data from the Actical will be downloaded when participants first enter the laboratory and if participants do not adhere to the aforementioned criteria, they will be excluded from study participation.

The Whoop strap is a new device we are implementing in this protocol to compare to recordings of sleep from the Actical. The Whoop strap is a wrist-worn device that provides measures of heart rate, heart rate variability and sleep staging derived from actigraphy and photoplethysmography. Participants will wear the Whoop bands as an additional measure of heart rate and sleep quality on their dominant hand. This device will be worn by participants to assess sleep but will not be used for screening purposes.

\section{Sedentary behaviour}

Sedentary behaviour will be assessed during the week prior to study commencement via an activPAL (PAL Technologies, Glasgow, Scotland) accelerometer worn continuously on the anterior midline of the thigh. ${ }^{78}$ The device, weighting $9 \mathrm{~g}$ is approximately $24 \times 43 \times 5 \mathrm{~mm}$ in size and is housed in a waterproof nitrile sleeve. The device will be attached to the participants thigh via a standard adhesive dressing and worn continuously throughout the 7-day pre-study period. The activPAL is a triaxial accelerometer capable of determining activity duration, step count, sit-to-stand transitions and time spent in sitting/lying based on acceleration information. Data will be sampled at $40 \mathrm{~Hz}$ and analysed in $15 \mathrm{~s}$ epochs, as standard in the literature. ${ }^{79}$ This device is known to be valid in laboratory environments and measures sedentary time, physical activity and posture (sitting, lying down, standing and stepping). ${ }^{80-83}$ This device will also be used to objectively measure participants' normal levels of sedentary behaviour and corroborate that participants have adhered to the instruction of avoiding moderate-vigorous-intensity exercise. Participants will be excluded if they have not adhered to this guideline.

\section{Experimental procedure \\ Laboratory setting}

The Appleton Institute Sleep Laboratory in Adelaide (Australia) comprises of a sound attenuated and 


\section{Dayshift Conditions}

(i) Sitting and 9-h sleep opportunity

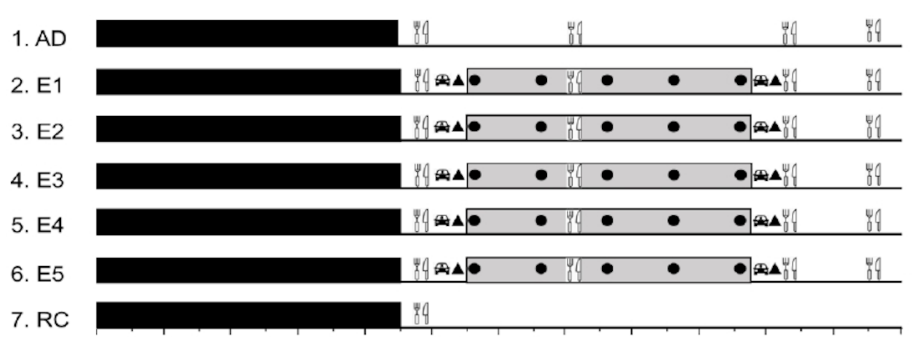

(iii) Sitting and 5-h sleep opportunity

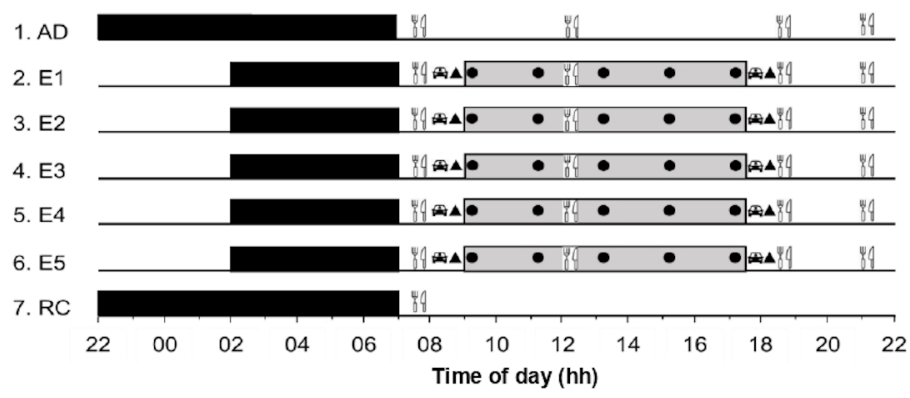

(ii) Breaking up sitting and 9-h sleep opportunity

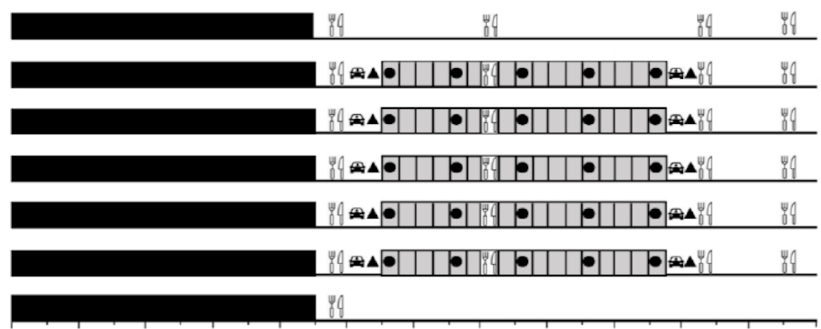

(iv) Breaking up sitting and 5-h sleep opportunity

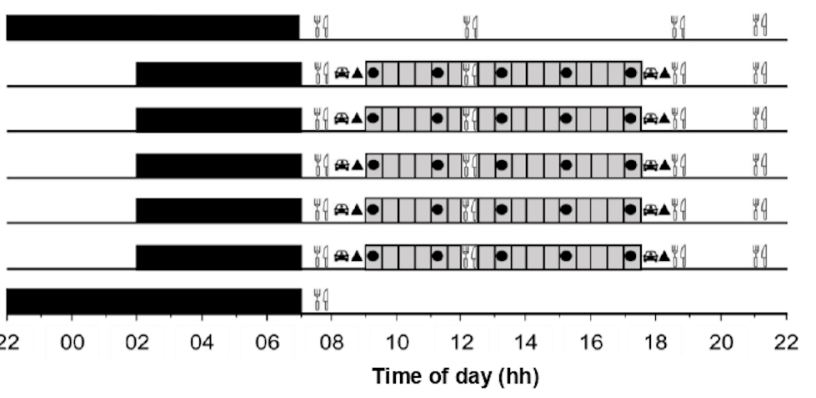

Legend: Sleep Opportunity

Wh Meal Driving Simulator

A Karolinska Drowsiness Test - Cognitive and self-perceived testing battery

Figure 2 Dayshift experimental design and protocol for the four experimental conditions: (i) Sitting and 9-h sleep opportunity, (ii) Breaking up sitting and 9-h sleep opportunity, (iii) Sitting and 5-h sleep opportunity, (iv) Breaking up sitting and 5-h sleep opportunity, across time of day (hh; $\mathrm{x}$-axis) and day of study (y-axis). AD, Adaptation Day; E1 to E5, Experimental Days; RC, Recovery Day.

temperature-controlled $\left(21^{\circ} \mathrm{C} \pm 2^{\circ} \mathrm{C}\right)$ sleep research laboratory. There are six bedrooms, each with a king-size single bed and ensuite, and two separate living room/ kitchen facilities. Consistent with the pilot study, ${ }^{24}$ during wake periods, light levels will be maintained at $>100$ lux, reflecting typical daily light levels. ${ }^{84}$ Dim light conditions $(<10$ lux $)$ will be employed between 08:00 to 12:00 in all nightshift conditions, to reflect dimmer working conditions at night and to reduce the phase shifting effects of bright light. ${ }^{85}$ Dim light (<10 lux) will also be employed for between 22:00 to 00:00. During all sleep periods, in all conditions, light levels will be negligible $(<0.3$ lux $)$.

\section{Procedure}

Participants will live in the Appleton Institute Sleep Laboratory for seven consecutive days and nights. The full experimental protocols are shown for dayshift conditions (figure 2) and nightshift conditions (figure 3). These days include one arrival evening, one Adaptation Day (AD), five Experimental Days (E1 to E5) and one Recovery Day (RC). On the arrival evening, participants will present at the laboratory at 17:00. Participants will be briefed on the study procedures and provided with the opportunity to ask any further questions. All participants will then have an Adaptation sleep from 22:00 to 07:00. On the Adaptation Day, participants will be familiarised with all relevant questionnaires and all components in the cognitive and self-perceived capacity battery. Participants who are allocated to nightshift conditions nap from
15:00 to 17:00 on the Adaptation Day, in preparation for their first nightshift.

On E1 to E5 participants will complete simulated work shifts (8.5 hour in duration). The simulated work shifts will occur from 09:00 to 17:30 in the dayshift conditions (figure 2) and from 22:00 to 06:30 in the nightshift conditions (figure 3). Immediately prior to and following each simulated work shift, participants will complete a simulated commute (a 20 min driving task, described below). During work shifts, participants will be required to remain seated at all times, aside from designated breaking up sitting periods. During sitting periods participants can engage in seated activities such as reading, watching television or other quiet activities.

Cognitive performance and self-perceived capacity will be assessed in testing batteries five times per Experimental Day. These will be performed at 09:00, 11:00, 13:00, 15:00 and 17:00 in dayshift conditions and 22:00, 00:00, 02:00, 04:00 and 06:00 in nightshift conditions.

During participant work shifts (E1 to E5), participants in the breaking up sitting condition will spend $3 \mathrm{~min}$ periods every $30 \mathrm{~min}$ performing light-intensity physical activity. This exercise will consist of walking at a speed of $3.2 \mathrm{~km} \cdot \mathrm{h}^{-1}$ on a motorised treadmill (Healthrider H95T; Icon Health \& Fitness Inc, Utah, USA) with a level incline $\left(0 \%\right.$ gradient) ${ }^{70} 71$ This duration, speed and frequency ${ }^{86}$ aligns with the breaking up sitting literature, representing light-intensity exercise. ${ }^{28} 70-728788$ For participants in the dayshift condition, breaking up sitting will occur every 


\section{Nightshift Conditions}

(i) Sitting and 9-h sleep opportunity

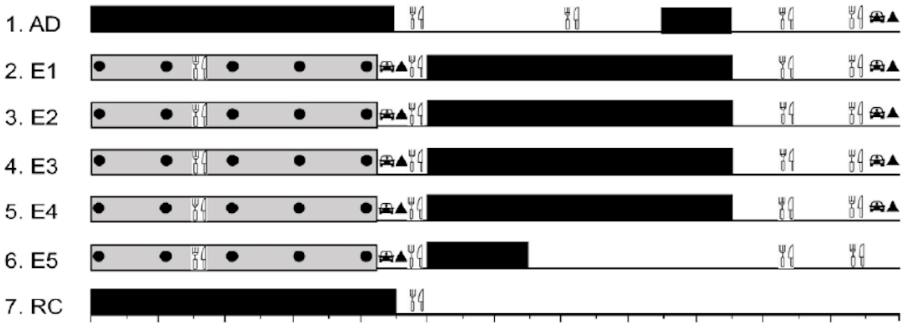

(iii) Sitting and 5-h sleep opportunity

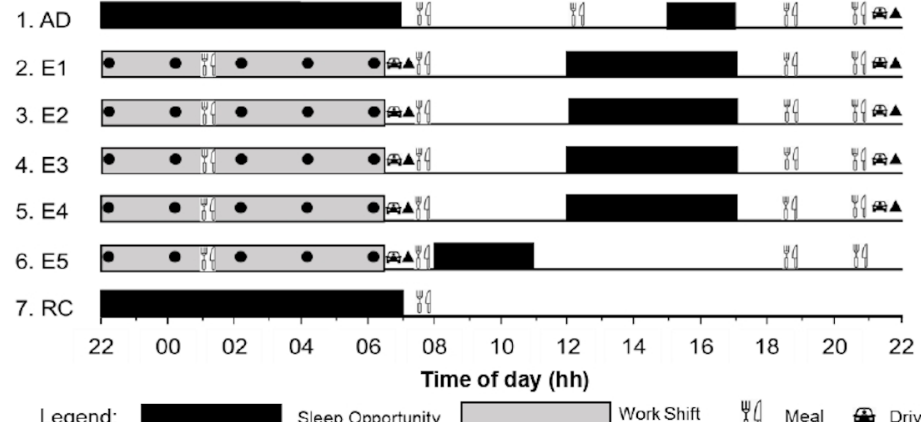

Legend:

Figure 3 Experimental design and protocol for the four nightshift experimental conditions: (i) Sitting and 9-h sleep opportunity, (ii) Breaking up sitting and 9-h sleep opportunity, (iii) Sitting and 5-h sleep opportunity, (iv) Breaking up sitting and 5-h sleep opportunity, across time of day (hh; $x$-axis) and day of study (y-axis). AD, Adaptation Day; E1 to E5, Experimental Days; RC, Recovery Day.

$30 \mathrm{~min}$ from 09:00 to 17:00, and for nightshift participants from 22:00 to 06:00. In total, participants in the breaking up sitting conditions will perform $17 \times 3 \mathrm{~min}$ bouts of exercise per Experimental Day (51 min in total). All participants in the sitting condition will remain seated for the entire study, aside from walking to and from the dining room at meal-time (distance: $32 \mathrm{~m}$ ) and to use the bathroom (distance: $8 \mathrm{~m}$ ). Participants in the breaking up sitting condition will do the same, except for designated breaking up sitting periods.

Sleep opportunities will be 5 hour or 9 hour depending on condition and will either occur during the night (dayshift conditions) or during the day (nightshift conditions), see table 5. Sleep parameters will be assessed using polysomnography for each sleep period.

Participants in nightshift conditions will also have a nap opportunity from 08:00 to 11:00 after their final nightshift. For all participants, the final night in the laboratory will be a recovery night with a 9 hour sleep opportunity (22:00 to 07:00). Participants will shower and eat breakfast on the Recovery Day, participants will be given a taxi voucher to travel home (approximately 08:30).

Table 5 Timing of sleep opportunities in the dayshift and nightshift condition

\begin{tabular}{lll}
\hline & $\begin{array}{l}\text { 9hour sleep } \\
\text { opportunity }\end{array}$ & $\begin{array}{l}\text { 5hour sleep } \\
\text { opportunity }\end{array}$ \\
\hline Dayshift & $22: 00$ to $07: 00$ & 02:00 to 07:00 \\
\hline Nightshift & 08:00 to 17:00 & 12:00 to 17:00 \\
\hline
\end{tabular}

\section{Outcome measures}

\section{Control measures}

Several control variables will be collected during this protocol that may each independently affect the primary and secondary outcomes of this study.

\section{Body weight}

Body weight will be measured daily (prior to the start of the work shift) via a standard scale (Salter, Model 9102, Thebarton, South Australia) using the same procedure as the screening protocol to ensure that participants weight is not changing significantly during the study. For participants in the dayshift condition, this will occur at 07:15. Nightshift participants will be weighed at 21:45.

\section{Physical activity monitoring}

ActivPAL monitors (PAL Technologies, Glasgow, Scotland) as described previously, will be worn for the duration of the study. These devices will be worn to measure physical activity during breaking up sitting periods, in addition to all other incidental activity occurring during the study (eg, bathroom and meal breaks).

\section{Activity monitor to measure sleep}

Activity monitors (Actical MiniMitter/Respironics, Bend, Oregon) and the Whoop (CB Rank, Greater Boston, New England, USA), as described above, will also be worn for the duration of the study (a) to corroborate sleep/ wake times, (b) as a contingency for polysomnographic measures and (c) for potential validation studies. Sleep 
periods will be scored using the same procedure as used in prescreening.

\section{Food}

Meals consistent with a standard Western diet will be provided for participants throughout the study. Daily macronutrient profile will consist of approximately $26 \%$ protein, $20 \%$ fat and $54 \%$ carbohydrate. To ensure that the food provided in during the protocol is consistent across participants, those with food allergies, dietary requirements or a vegan diet will be excluded. Daily estimated energy requirements (EER) will be calculated using a modified Harris Benedict equation. ${ }^{89}$ For those in the breaking up sitting condition, an activity factor of 1.5 will be applied, standard in studies with increased physical activity. ${ }^{28}$ For those in the sitting condition, an activity factor of 1.3 will be applied, standard in sleep studies with extreme sitting conditions. ${ }^{90} 91$ Further, to account for the $1 \%$ per $\mathrm{h}$ increase in total daily energy expenditure with sleep restriction, ${ }^{92}$ energy intake for those in the sleep restriction condition will be increased by $4 \%$ for the 4 hour of sleep restriction ( 5 hour sleep opportunity instead of the 9 hour sleep opportunity).

On all Adaptation Days, Recovery Days and for dayshift participants, meals will occur at 07:20 (breakfast; 15\% of EER), at 12:10 (lunch; 35\% of EER) and at 18:30 (dinner; $25 \%$ of EER). A snack will be provided at 21:00 (25\% of EER). Participants in the nightshift condition will consume meals at 07:20 (breakfast; 25\% of EER), at 18:30 (dinner; 25\% of EER) and at 01:30 (lunch; 25\% of EER), with a snack at 21:00 (25\% of EER). Participants will be encouraged to consume $100 \%$ of all meals and snacks, with any leftover food weighed and recorded by research staff.

\section{Primary outcome measures}

\section{Physiological measures}

\section{Continuous glucose monitoring system}

Participants will have an interstitial glucose monitoring device (MiniMed Medtronic Australasia PTY, Adelaide, Australia) fitted by a trained research assistant. This lightweight, portable, minimally invasive system consists of a sterile, single use glucose oxidase-based electrode sensor $(0.6 \mathrm{~mm}$ gauge $\times 12.7 \mathrm{~mm})$ and a matchbox sized electronic monitor that records and stores data. The tiny, hair-like, flexible sensor will be inserted just under the skin of the abdomen (in the subcutaneous tissue) using a spring-loaded device. The sensor is held in position using an adhesive dressing. The sensor will be inserted on the morning of the Adaptation Day and removed on the Recovery Day.

The device will be calibrated every 5 hour via capillary finger pricks, using a lancing device (Accu-Chek Softclix Lancing Device LD01, Roche Diabetes Care Inc, USA), glucose electrode test strips (Optium, Roche Diabetes Care Inc, USA) and a blood glucose meter (Optium Xceed, Roche Diabetes Care Inc, USA). This is a standard measurement technique used in sleep laboratory studies where glucose concentration measurement is required. ${ }^{93}$ This system has also been validated against plasma glucose. ${ }^{94}$ The trapezoidal method will be used to derive the total area under the interstitial glucose response curve from the post-prandial blood glucose measurements. Raw interstitial glucose values will be used to calculate daily minimum, maximum and mean interstitial glucose concentrations. Data will be downloaded from the glucose monitoring device using Medtronic CareLink Pro.

\section{Blood pressure}

Blood pressure is a standard marker of cardiovascular health and is known to be influenced by breaking up sitting in the previous studies. ${ }^{95}$ Blood pressure will be measured via an ambulatory blood pressure monitor (TM-2430 Ambulatory Blood Pressure Monitor, A\&D Australasia Pty Ltd, Thebarton, South Australia). On the Adaptation Day, participants will be given verbal instructions regarding the frequency of measurements (ie, blood pressure cuff inflations and deflations), how to manually begin a blood pressure reading when instructed by the researcher and to stay seated and still during recordings. Blood pressure cuffs will be fitted to ensure the correct size cuff for each participant, on the participants non-dominant arm. Blood pressure monitors will be programmed to begin automatic recordings every $30 \mathrm{~min}$ on Experimental Days 1, 3 and 5 during the work shift (worn continuously from 09:00 to 17:00 for dayshifts and 22:00 to 06:30 during nightshifts). This corresponds to a recording taken $5 \min ^{28} 95$ prior to exercise commencement in the breaking up sitting condition. Post-exercise recordings will also be taken immediately on finishing breaking up sitting bouts for the participants in the breaking up sitting condition. Despite no exercise being performed, participants in the sitting condition will also perform a blood pressure recording at this time. Participants will be instructed to keep their arm still and at heart level during recording periods. ${ }^{96}$ Data will be downloaded from the ambulatory blood pressure monitor using Doctor Pro 3 blood pressure analysis software (A\&D Australasia Pty Ltd, Thebarton, South Australia).

\section{Heart rate}

Heart rate will be monitored via a standard heart rate monitor worn around the chest (Polar HR M400). The heart rate monitor will be worn on Experimental Days 1, 3 and 5, during the work shift (worn continuously from 09:00 to 17:00 for dayshifts and 22:00 to 06:30 during nightshifts). Heart rate is a marker of cardiovascular health and will be used to determine the acute cardiovascular response to the breaking up sitting periods. ${ }^{97}$ Data will be downloaded using Polar FlowSync software.

Cognitive performance and self-perceived capacity testing battery The cognitive performance and self-perceived capacity testing battery will last for approximately $25 \mathrm{~min}$ (figure 4 ). 


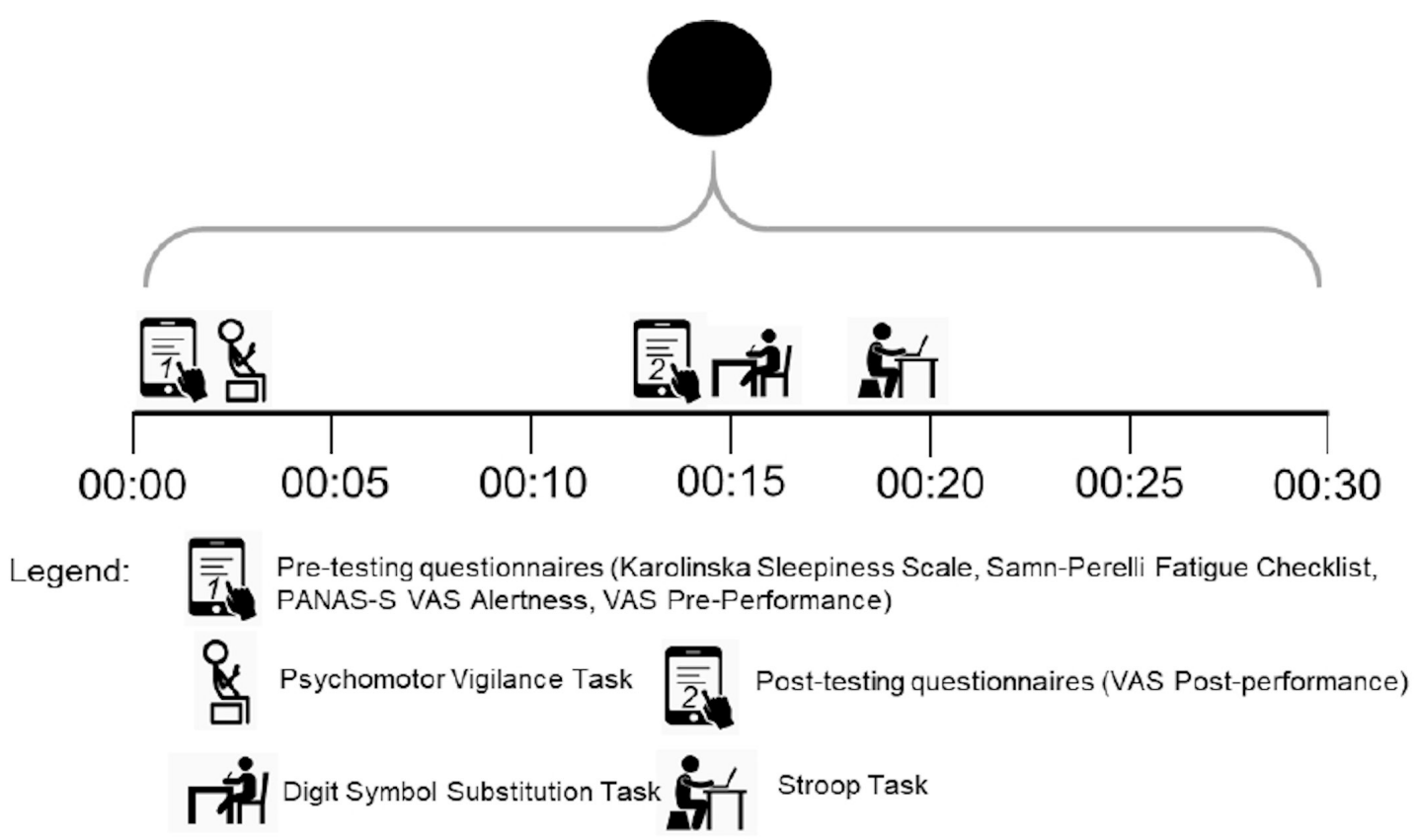

Figure 4 Protocol of the cognitive performance and self-perceived capacity testing battery. PNAS - S, Positive and Negative Affect Schedule - Sleep; VAS Alertness, Visual Analogue Scale of Alertness; VAS Pre-Performance, Visual Analogue Scale PrePerformance; VAS Post-Performance, Visual Analogue Scale Post-Performance. This testing battery is completed for all dayshift conditions at 09:00, 11:00, 13:00, 15:00 and 17:00, and in all nightshift conditions at 22:00, 00:00, 02:00, 04:00 and 06:00.

The pre-testing and post-testing questionnaires (i to $\mathrm{v}$ below) will be presented via Qualtrics (Qualtrics, Provo, Utah, USA) using an Apple iPad (Apple Inc, Cupertino, California, USA). Data from Qualtrics will be downloaded and double-entered. The Psychomotor Vigilance task is performed using a handheld device, the Digit Symbol Substitution task is a pen and paper task and the Stroop task is performed on a computer.

\section{Karolinska Sleepiness Scale}

The Karolinska Sleepiness Scale is a well validated 1-item questionnaire that requires respondents to rate their current level of sleepiness on a 9-item scale. This scale goes from 1 ('extremely alert') to 9 ('very sleepy, great effort to keep awake, fighting sleep').$^{98}$

\section{Samn-Perelli Fatigue Scale}

The Samn-Perelli Fatigue Scale will be completed. This 7-point scale measures fatigue and requires participants to select which item most reflects their current level of fatigue. Options go from 1 ('fully alert, wide awake') to 7 ('completely exhausted, unable to function effectively'). This scale is well-validated to measure fatigue. ${ }^{99}$

\section{Positive and Negative Affect Schedule - Sleep}

The Positive and Negative Affect Schedule - Sleep (PANAS-S) is a shortened version of the validated Positive and Negative Affect Schedule ${ }^{100}$ that measures specific feelings and emotions related to sleepiness and fatigue. The PANAS-S contains 14 feelings or emotions (such as energetic, content and attentive). Participants are required to use a 5-point scale anchored with 1 representing 'very slightly or not at all' and 5 representing 'extremely' to indicate the extent participants are experiencing that feeling or emotion in the moment.

\section{Visual Analogue Scales - Alertness}

A 100-point Visual Analogue Scale (VAS) will be used to quantify subjective alertness. Participants are asked 'how alert do you feel right now?'. In response to this question, participants will move the slider cursor to a point between 0 ('not at all alert') and 100 ('extremely alert'). Scores are integers between 0 and 100 (ie, no decimal places are used for scoring), with higher scores reflecting greater alertness. ${ }^{101}$

\section{Visual Analogue Scales - Performance}

Prior to and following (pre and post) the completion of the psychomotor vigilance task (PVT), participants will complete VAS asking them to rate their perceived performance on the PVT. Prior to completing the PVT, participants will respond to the question 'How well do you think you will perform?'. Following completion of the PVT, participants will respond to the question 'How well did you think you performed?'. The slider cursor will move between 0 ('extremely poorly') and 100 ('extremely well'). Scores will be integers between 0 and 100 (ie, no decimal places are used for scoring). Higher scores reflect better perceived performance on the PVT.

\section{Psychomotor vigilance task}

A 10 min PVT will be performed (PVT-192, Ambulatory Monitoring Inc, Ardsley, New York). ${ }^{102}$ The PVT is the gold standard measure of fatigue-related cognitive performance and is used widely in the sleep and fatigue literature. ${ }^{103}$ This task involves participants pressing a 
button on a handheld device as quickly as possible in response to a stimulus. Participants will complete the task while seated in a chair facing a wall (seated approximately $1 \mathrm{~m}$ away from the wall). Participants are instructed to sit straight and to keep both feet on the ground, while holding the device in both hands. Stimuli are presented at random intervals between 2 and $10 \mathrm{~s}$ for the duration of the task. Participants will complete three 10 min PVTs on the Adaptation Day, to account for any learning effects. ${ }^{104}$ The PVT measures sustained attention, vigilance and reaction time, and has been highly correlated with sleep loss and circadian misalignment. ${ }^{105-107}$ Outcome measures include lapses of more than $500 \mathrm{~ms}$, reciprocal reaction time (RRT), mean fastest $10 \%$ of reaction time (RT) and mean slowest $10 \%$ of RRT. ${ }^{108}$

\section{Digit symbol substitution test}

The digit symbol substitution test (DSST) is a task that assesses working memory, response speed and visuomotor coordination. ${ }^{109}$ Participants are provided with a key consisting of nine digits (0 to 9 ) which are each associated with unique predetermined symbols. Participants are given a series of randomly ordered digits and are required to correctly draw the associated symbols for each digit. Participants will be given 90 s to complete as many symbols as possible and are not permitted to skip any digits. Participants will complete 14 familiarisation trials $\mathrm{AD}$ to avoid any learning effects. The outcome variable of the DSST is the number of correct responses (accurate and legible) completed in the $90 \mathrm{~s}$. All DSSTs will be scored by the same researcher and then double-entered.

\section{Stroop task}

The Stroop task ${ }^{110}$ is a test of response inhibition to cognitive interference. ${ }^{11}$ The Stroop task has been previously shown to be sensitive to sleep restriction ${ }^{112} 113$ and acute bouts of physical activity. ${ }^{114}$ This task consists of a 2 min test requiring participants to respond to the word presented on the screen via the corresponding coloured sticker placed on an alphanumeric keypad. The usual Stroop task requires participants to respond to the font colour, however in this task participants had to read the word and respond to the colour word that is spelled out by pressing the corresponding key on a keypad. This removes the opportunity for participants to use a strategy where they avoid looking at the screen and view the colour of the word using their peripheral vision. ${ }^{115}$ In the current study, there are two conditions, a matching condition, in which the colour word is written in the corresponding font colour (eg, 'RED' is presented in red font), and a non-matching condition in which the colour word is written in a non-corresponding font colour (eg, 'RED' is presented in green font). Participants are required to respond to the colour word that is spelled out, rather than the font colour of the word. Colour-word displays are either red, blue, green or yellow, and will be presented on a standard 17-inch computer screen. The variables for analysis will include percentage correct and mean reaction time (ms) for matching and non-matching words.

\section{Secondary outcome measures}

Sleep

\section{Objective sleep monitoring - polysomnography}

In this study all sleep opportunities are prescribed (naps and main sleep periods). However, breaking up sitting can impact the structure of sleep (eg, sleep stages), as seen in our pilot study. ${ }^{24}$ Therefore, sleep outcomes will be used as a covariate when answering some research questions, and an outcome measure for other research questions. All sleep periods will be measured using polysomnography (PSG), the gold standard measurement technique for determining total sleep time (TST). TST includes the total amount of sleep (minutes) obtained between bed and rise times. A standard electroencephalography (EEG) montage as measured by the Compumedics Grael PSG/ EEG system (Melbourne, Australia) will be used. Recordings will be taken from the three standard electrode channels, $\mathrm{C} 3 / \mathrm{M} 2, \mathrm{~F} 4 / \mathrm{M} 1$ and $\mathrm{O} 2 / \mathrm{M}^{116}$ in addition to electromyography recordings, from the left, right and middle of the jawline and electrooculography recordings (left and right outer canthi). Sleep records will be blinded and scored by a trained sleep technician in 30 s epochs according to standard criteria. ${ }^{116}$ Variables include total sleep time, sleep efficiency, sleep onset latency, wake after sleep onset, stage N1, stage N2, stage N3 (slow wave sleep) and stage REM (rapid-eye movement).

\section{Pre-sleep and post-sleep questionnaires}

Pre-sleep and post-sleep questionnaires will be completed using Qualtrics (Qualtrics, Provo, Utah, USA), presented using an Apple iPad (Apple Inc, Cupertino, California, USA). Completed questionnaires will be downloaded and double-entered into a spreadsheet. The pre-sleep questionnaire includes the one-question Karolinska Sleepiness Scale, ${ }^{117}$ and the question 'How well do you expect to sleep tonight?', with answers ranging from 1 (very poorly) to 5 (very well). Seven 100-point Visual Analogue Scales will also be completed. These scales are used to measure: hunger ('How hungry were you today before each meal?' anchored by 0 'Not at all hungry' and 100 'as hungry as I've ever felt'), stomach upset ('How much stomach upset did you experience today?' anchored by 0 'no upset stomach' and 100 'extreme upset stomach'), bloating ('How much bloating did you experience today?' anchored by 0 'I did not feel bloated at all and 100 'I feel extremely bloated'), stress ('How stressed were you today?' anchored by 0 'not at all stressed' and 100 'extremely stressed'), concentration ('Did you experience problems concentrating today?' anchored by 0 'absolutely not' and 100 'extreme problems concentrating'), physical activity ('Did you feel physically active today?' anchored with 0 'absolutely not' and 100 'extremely active') and mental activity ('Did you feel mentally active today?' anchored with 0 'absolutely not' and 100 'extremely active'). 
The post-sleep questionnaire includes 10 questions, including the Karolinska Sleepiness Scale, 'How was your sleep?' with answers ranging from 1 (very poor) to 5 (very good), 'How restless was your sleep?' ranging from 1 (very restless) to 5 (calm), 'Did you wake before being woken by a researcher?' ranging from 1 (yes, woke up much too early) to 5 (no), 'How difficult did you find it to fall asleep?' ranging from 1 (very difficult) to 5 (very easy), 'Do you feel well-rested?' ranging from 1 (not rested at all) to 5 (completely rested), 'Did you get enough sleep?' ranging from 1 (no, definitely too little) to 5 (yes, definitely enough) and open-ended questions asking participants 'How long did it take you to fall asleep?', 'How much sleep do you think you obtained?' and 'How many times did you wake up during the night?' Participants will also be asked to provide any additional comments regarding their sleep that they think are relevant.

\section{Simulated commute}

Before and after each designated work shift, all participants will complete a simulated commute. This consists of pre-drive questionnaires, Activation-Deactivation Adjective Check List (AD ACL), a 20 min driving simulator task and a post-drive questionnaire (NASA Task Load Index (TLX).

\section{Pre-drive Visual Analogue Scales}

Pre-drive Visual Analogue Scales will be presented to participants prior to each driving simulator task. A 100point VAS will be presented to participants using the Qualtrics platform (Qualtrics, Provo, Utah) presented using an Apple iPad (Apple Inc, Cupertino, California, USA). The participants will be asked three questions. The first two questions ask participants to rate on the slider scale 'How good do you think you will be at keeping to the speed limit?' and 'How good do you think you will be at keeping the car in the middle of the left lane?'. Both of these questions are anchored at 0 representing 'not good at all' and 100 representing 'extremely good'. The last question asks 'How confident do you feel in your ability to drive safely in this drive?', this is anchored at 0 for 'not very confident' and 100 for 'very confident'. Completed questionnaires will be downloaded and double-entered.

\section{Activation-Deactivation Adjective Check List}

The AD ACL will be completed prior to the driving simulator task. This checklist is a widely used, rapid and easy to understand self-assessment of cognitive arousal and activation levels. ${ }^{118} 119$ The questionnaire presents participants with 20 adjectives which can be rated from the four given answer categories; 'definitely feel', 'feel slightly', 'cannot decide' and 'definitely do not feel'. ${ }^{118}$ This questionnaire is pen and paper-based and will be scored by a researcher then double-entered.

\section{Pre-drive and post-drive Visual Analogue Scales}

The post-drive Visual Analogue Scales will be presented to participants using the Qualtrics platform (Qualtrics, Provo, Utah) presented using an Apple iPad (Apple Inc,
Cupertino, California, USA). These questions allow the participants to reflect on their performance by asking similar questions to the pre-drive questions, but in the past tense with the same anchor points. The first question asks participants 'How good do you think you were at keeping to the speed limit during the drive?' and 'How good do you think you were at keeping the car in the middle of the left lane during the drive?'. The last question asks participants 'How confident do you feel now in your ability to drive safely?'. Completed questionnaires will be downloaded and double-entered.

\section{Driving simulator}

Driving performance will be measured using the York Driving Simulator at the start and end of each participant's work shift (York Computer Technologies, Kingston, Ontario). The driving simulation will be presented on a single computer screen which displays a forward-facing view from the driver's seat. Each participant's room will be equipped with a standard computer screen with steering wheel and pedal attachments connected and placed in the appropriate position (Logitech, Lausanne, Switzerland). Participants will complete a $20 \mathrm{~min}$ driving task consisting of a simulated daytime rural drive with a speed limit of $110 \mathrm{~km} \cdot \mathrm{h}^{-1}{ }^{29}$ The highway scenario is comprised of a single carriageway, two lane road with traffic driving in both directions. ${ }^{29}$ Participants will be instructed to obey standard Australian road rules.

The $20 \mathrm{~min}$ drive will be binned into four $5 \mathrm{~min}$ bins to allow for time on task analyses. Driving performance will be measured using speed variability and lane variability. ${ }^{29}$ Lane position refers to the location of the car within the lane and is measured in metres from the left side of the road up to 25 times per second to calculate the number of violations. ${ }^{91}$ Speed variability will be recorded in $\mathrm{km} . \mathrm{h}^{-1}$ to indicate average speed variability. Greater speed variability is indicative of decreased driving performance. ${ }^{91}$ These measures have been used in several previous studies into simulated driving in conditions of shiftwork or sleep restriction studies. ${ }^{29} 91120$

\section{NASA Task Load Index}

The NASA TLX is a well-established subjective questionnaire which assesses perceived workload demand for a given task. ${ }^{121}$ The NASA TLX assesses workload across six subscales; Mental Demand, Physical Demand, Temporal Demand, Performance, Effort and Frustration. ${ }^{119}$ Participants completed this assessment via the NASA TLX software application (NASA, Washington DC, Washington) on a provided Apple iPad (Apple Inc, Cupertino, California, USA). Participants will be presented with 15 questions which compared the six subscales and asked to circle which subscale was most influential during the driving task. ${ }^{122}$ Participants are then be asked to indicate on a sliding scale how they subjectively experienced each of the six subscales. ${ }^{123}$ The sliding scale is divided into 20 equal portions which are labelled at either end of the scale with 'low' or 'high' and 'perfect' or 'failure'. ${ }^{124}$ Data 
from the software application will be double-entered into a spreadsheet.

\section{Karolinska drowsiness test}

The Karolinska drowsiness test (KDT) is a standardised recording of a predefined amount of waking EEG under controlled experimental conditions with specific periods where participant's eyes are open and closed.$^{98}$ The KDT will be performed after each simulated drive (08:40 and 18:00 for dayshift participants and 21:40 and 07:00 for nightshift participants), to identify drowsiness levels while driving. Participants will sit upright in a comfortable chair located in their room with standard ambient room lighting ( 100 lux). During the KDT, participants will be asked to look at a marker (black dot) which is fixed on the wall at eye level at a distance of 1 metre and will be instructed to relax and keep as still as possible for $2.5 \mathrm{~min}$. Participants will then be asked to close their eyes while remaining seated in the same position for an additional $2.5 \mathrm{~min}$. Finally, another $2.5 \mathrm{~min}$ of waking EEG will be recorded while the participant's eyes are open. This is consistent with standard KDT procedure in the Appleton Institute Sleep Laboratory. ${ }^{125}$

\section{Standard questionnaires}

On the Adaptation Day, participants will complete several questionnaires assessing behavioural, psychological and sociodemographic characteristics to allow us to describe participants sample characteristics at baseline. These questionnaires will be presented to participants using the Qualtrics platform (Qualtrics, Provo, Utah) on an Apple iPad (Apple Inc, Cupertino, California, USA). Completed questionnaires will be downloaded and double-entered.

\section{Active Australia Survey}

The Active Australia Survey collects information on the frequency and duration of varying types of physical activities undertaken, and was developed for use in Australian adults aged 18 to $65 .{ }^{126}$ Participants are asked to reflect on their engagement with the following activities across the last week: walking, gardening, vigorous-intensity physical activity and moderate-intensity physical activity. The Active Australia Survey has been used in Australian national and cohort surveys ${ }^{127}$ and is primarily used for health surveillance in Australia. ${ }^{126}$

\section{Dietary Guideline Index}

The Dietary Guideline Index (DGI-2013) includes 13 questions relating to the types of foods that are generally consumed. ${ }^{128}$ Questions require participants to indicate which types of certain food groups they would typically choose. For example, participants are asked to indicate which type of bread they generally consume (ie, high fibre white bread, white bread, wholemeal bread, rye bread, whole/multigrain bread or no bread). Similar questions are also included, regarding the consumption of fruits, vegetables, milk, fat-based spreads, meat, rice/ pasta/noodles and other dietary components.

\section{Eysenck Personality Inventory}

The Eysenck Personality Inventory is a standardised questionnaire designed to categorise participants by key personality traits. ${ }^{129}$ This questionnaire consists of 57 yes/ no response items, where participants are asked questions such as 'do you often long for excitement?' and 'do you find it very hard to take no for an answer?'. Items are used to determine scores on extraversion and neuroticism scales, based on responses to certain questions (ie, questions are designed to determine either extraversion or neuroticism)..$^{130}$ This questionnaire also comprises a 9-item 'lie scale' to provide an index of validity and to increase test-retest reliability. ${ }^{131}$

\section{Locus of Control questionnaire}

The Locus of Control questionnaire includes 29 items with binary choices ${ }^{132}$ and is used to determine whether the participant has a primary belief in internal or external control of events in their lives. Participants are required to choose which statement they agree with. Statements include, for example, (a) 'People are lonely because they don't try to be friendly' and (b) 'There's not much use in trying too hard to please people, if they like you, they like you'. Based on participants responses to each question, they are either allocated a score of 0 or 1 . These scores are then summed to provide the overall Locus of Control score. Higher scores are reflective of internal loci of control. ${ }^{132}$

\section{Munich Chronotype Questionnaire}

The Munich Chronotype Questionnaire (MCTQ) includes four questions regarding the timing of bed, sleep and wake times on work and non-workdays to determine chronotype. ${ }^{133}$ The MEQ (another measure of chronotype) is completed by participants during the screening process, and collects information regarding the midpoint between sleep onset and wake time on non-workays allowing for a comparison of early and late chronotypes within the sample relative to other participants. ${ }^{134}$ The MCTQ will be completed during the study protocol as a further measure of chronotype as it collects additional detailed information regarding chronotype by determining the midpoint between sleep onset and wake time on non-workdays which allows for the comparison of early and late chronotypes within the sample relative to other participants. ${ }^{134}$

\section{Sleep Need Questionnaire}

The Sleep Need Questionnaire includes six questions designed to elicit information about participants sleep need. Participants will be asked how much sleep they typically obtain, in addition to how much sleep they believe they need to function at peak (physically and mentally).

\section{State Trait Anxiety Inventory}

The State Trait Anxiety Inventory x-2 will assess trait anxiety. ${ }^{135}$ This questionnaire includes items such as 'I feel pleasant' or 'I wish I could be as happy as others 
seem to be'. Participants are asked to respond in terms of how they generally feel. Items are scored from 1 ('almost never') to 4 ('almost always'). Based on standard practice, positive items will be reverse coded.

\section{STATISTICAL ANALYSIS}

All data will be stored in a de-identified format. Paper questionnaires will be stored in a locked filing cabinet and all computer-based data will be stored on password protected computer drives. Statistical analyses will be conducted using the latest SPSS software (V.26.0 software, IBM Corp, Armonk, New York). For each research question, a series of mixed models will be conducted to examine between group differences in cardiometabolic outcomes and cognitive performance. Models will have fixed effects including, activity (sitting or breaking up sitting), sleep (5 hour or 9 hour sleep opportunity), shiftwork (dayshift or nightshift), study day (2 to 6), sampling time point and a random effect of participant. Sleep variables will be included as covariates if significant differences arise between conditions (other than those implemented by design, for example, a significant difference in time in bed between the 5 hour versus 9 hour sleep opportunity). Gender and body mass index will also be included as covariates in all analyses. Significant effects will be further investigated by within-subjects post hoc comparisons. Residuals will be checked for normality and inform the specification of family and link functions. Alpha will be set at 0.05 .

\section{PATIENT AND PUBLIC INVOLVEMENT}

The design of this research was done without patient or public involvement, and the conduct of this research will be carried out without the involvement of patients (participants). Members of the public will be involved in this research as participants. While patients and the public will not be invited to comment on the study design or interpret the results, we aim to widely disseminate our results to the public through journal articles, conference presentations and media releases. The findings of the study will be disseminated to participants on their request.

\section{ETHICS AND DISSEMINATION}

This study protocol was approved by the Central Queensland University Human Research Ethics Committee (CQUHREC) in September 2019 (0000021914). All participants will provide electronic informed consent prior to completing the first screening survey. We will seek ethical approval from the CQUHREC for any protocol modifications and these modifications will be updated on the AustralianNew ZealandClinical Trials Registry. All authors will have access to the final de-identified data set. The findings of this study will be disseminated via peer-reviewed publications and presentations at national and international conferences. Findings will also form part of student dissertations.

Author affiliations

${ }^{1}$ Appleton Institute, Central Queensland University - Adelaide Campus, Wayville, South Australia, Australia

${ }^{2}$ School of Health Medical and Applied Sciences, Central Queensland University, Rockhampton, Queensland, Australia

${ }^{3}$ School of Medicine \& Public Health, Faculty of Health and Medicine, The University of Newcastle, Callaghan, NSW, Australia

${ }^{4}$ Priority Research Centre for Physical Activity and Nutrition, The University of Newcastle, Callaghan, NSW, Australia

${ }^{5}$ Psychology Department, Swansea University, Swansea, United Kingdom ${ }^{6}$ Stress Research Institute, Department of Psychology, Stocklholm University, Stockholm, Sweden

Twitter Grace E Vincent @PhDsleepy, Charlotte C Gupta @cc_gupta, Madeline Sprajcer @msprajcer, Corneel Vandelanotte @corneelvdl and Mitch J Duncan @ mitchjduncan

Contributors Conceptualisation: GEV, CV, MJD, PT and SF. Funding acquisition: GEV, CV, MJD, PT and SAF. Methodology: GEV, CCG, MS, CV, MJD, PT, ML, GT and SF. Writing —original draft: GEV, CCG and MS; Writing — review and editing: GEV, CCG, MS, CV, MJD, PT, ML, GT and SF.

Funding This research has received funding from the Australian Research Council (DP190101130). MJD is supported by a Career Development Fellowship (AP1141606) from the National Health and Medical Research Council

Competing interests None declared.

Patient and public involvement Patients and/or the public were involved in the design, or conduct, or reporting, or dissemination plans of this research. Refer to the Methods section for further details.

Patient consent for publication Not required.

Provenance and peer review Not commissioned; peer reviewed for ethical and funding approval prior to submission.

Open access This is an open access article distributed in accordance with the Creative Commons Attribution Non Commercial (CC BY-NC 4.0) license, which permits others to distribute, remix, adapt, build upon this work non-commercially, and license their derivative works on different terms, provided the original work is properly cited, appropriate credit is given, any changes made indicated, and the use is non-commercial. See: http://creativecommons.org/licenses/by-nc/4.0/.

ORCID iDs

Grace E Vincent http://orcid.org/0000-0002-7036-7823

Charlotte C Gupta http://orcid.org/0000-0003-2436-3327

Madeline Sprajcer http://orcid.org/0000-0002-4966-871X

Corneel Vandelanotte http://orcid.org/0000-0002-4445-8094

Mitch J Duncan http://orcid.org/0000-0002-9166-6195

Phil Tucker http://orcid.org/0000-0002-8105-0901

Michele Lastella http://orcid.org/0000-0003-1793-3811

Georgia A Tuckwell http://orcid.org/0000-0003-4607-7698

Sally A Ferguson http://orcid.org/0000-0002-9682-7971

\section{REFERENCES}

1 Australian Beureau of Statistics (ABS). Australian health survey: physical activity Canberra, 2011: 2.

2 van Uffelen JGZ, Wong J, Chau JY, et al. Occupational sitting and health risks: a systematic review. Am J Prev Med 2010;39:379-88.

3 Ding D, Lawson KD, Kolbe-Alexander TL, et al. The economic burden of physical inactivity: a global analysis of major noncommunicable diseases. The Lancet 2016;388:1311-24.

4 Adams RJ, Appleton SL, Taylor AW, et al. Sleep health of Australian adults in 2016: results of the 2016 sleep health Foundation national survey. Sleep Health 2017;3:35-42.

5 Hillman D, Mitchell S, Streatfeild J, et al. The economic cost of inadequate sleep. Sleep 2018;41:83.

6 House of Representatives Standing Committee on Health. Bedtime reading. Canberra, AUS: Commonwealth of Australia, 2019. 
7 Healy GN, Matthews CE, Dunstan DW, et al. Sedentary time and cardio-metabolic biomarkers in US adults: NHANES 2003-06. Eur Heart J 2011;32:590-7.

8 Schmid SM, Hallschmid M, Schultes B. The metabolic burden of sleep loss. Lancet Diabetes Endocrinol 2015;3:52-62.

9 Loh R, Stamatakis E, Folkerts D, et al. Effects of interrupting prolonged sitting with physical activity breaks on blood glucose, insulin and triacylglycerol measures: a systematic review and metaanalysis. Sports Med 2020;50:295-330.

10 Wilmot EG, Edwardson CL, Achana FA, et al. Sedentary time in adults and the association with diabetes, cardiovascular disease and death: systematic review and meta-analysis. Diabetologia 2012;55:2895-905

11 Cappuccio FP, D'Elia L, Strazzullo P, et al. Sleep duration and all-cause mortality: a systematic review and meta-analysis of prospective studies. Sleep 2010;33:585-92.

12 Katzmarzyk PT, Church TS, Craig CL, et al. Sitting time and mortality from all causes, cardiovascular disease, and cancer. Med Sci Sports Exerc 2009;41:998-1005.

13 Ekelund U, Tarp J, Steene-Johannessen J, et al. Dose-Response associations between accelerometry measured physical activity and sedentary time and all cause mortality: systematic review and harmonised meta-analysis. BMJ 2019;366:14570.

14 Ulf E, Jostein S-J, Brown Wendy J, et al. Does physical activity attenuate, or even eliminate, the detrimental association of sitting time with mortality? A harmonised meta-analysis of data from more than 1 million men and women. The Lancet 2016;388:1302-10

15 Ekelund U, Brown WJ, Steene-Johannessen J, et al. Do the associations of sedentary behaviour with cardiovascular disease mortality and cancer mortality differ by physical activity level? A systematic review and harmonised meta-analysis of data from 850 060 participants. Br J Sports Med 2019;53:886-94.

16 Chastin SFM, Palarea-Albaladejo J, Dontje ML, et al. Combined effects of time spent in physical activity, sedentary behaviors and sleep on obesity and cardio-metabolic health markers: a novel compositional data analysis approach. PLoS One 2015:10:10.

17 Larsen RN, Kingwell BA, Sethi P, et al. Breaking up prolonged sitting reduces resting blood pressure in overweight/obese adults. Nutrition, Metabolism and Cardiovascular Diseases 2014;24:976-82.

18 Bhammar DM, Sawyer BJ, Tucker WJ, et al. Breaks in sitting time: effects on continuously monitored glucose and blood pressure. Med Sci Sports Exerc 2017;49:2119-30.

19 Katzmarzyk PT. Standing and mortality in a prospective cohort of Canadian adults. Med Sci Sports Exerc 2014;46:940-6.

20 Vincent GE, Jay SM, Sargent C, et al. The impact of breaking up prolonged sitting on glucose metabolism and cognitive function when sleep is restricted. Neurobiol Sleep Circadian Rhythms 2018;4:17-23

21 Vincent GE, Jay SM, Sargent C, et al. Improving cardiometabolic health with diet, physical activity, and breaking up sitting: what about sleep? Front Physiol 2017;8:865.

22 Knutson KL. Sleep duration and cardiometabolic risk: a review of the epidemiologic evidence. Best Pract Res Clin Endocrinol Metab 2010;24:731-43.

23 St-Onge M-P, Grandner MA, Brown D, et al. Sleep duration and quality: impact on lifestyle behaviors and cardiometabolic health: a scientific statement from the American heart association. Circulation 2016;134:e367-86.

24 Vincent GE, Jay SM, Sargent C, et al. Does breaking up prolonged sitting when sleep restricted affect postprandial glucose responses and subsequent sleep architecture? - a pilot study. Chronobiol Int 2018;35:821-6.

25 Åkerstedt T, Wright KP. Sleep loss and fatigue in shift work and shift work disorder. Sleep Med Clin 2009:4:257-71.

26 Goel N. Neurobehavioral effects and biomarkers of sleep loss in healthy adults. Curr Neurol Neurosci Rep 2017;17:89.

27 Reynolds AC, Banks S, deprivation Tsleep. Chronic sleep restriction and sleep disruption. progress in brain research. Prog Brain Res 2020;185:91-103

28 Wennberg P, Boraxbekk C-J, Wheeler M, et al. Acute effects of breaking up prolonged sitting on fatigue and cognition: a pilot study. BMJ Open 2016;6:e009630.

29 Matthews RW, Ferguson SA, Zhou X, et al. Simulated driving under the influence of extended wake, time of day and sleep restriction. Accid Anal Prev 2012;45 Suppl:55-61.

30 Thorp AA, Kingwell BA, Owen N, et al. Breaking up workplace sitting time with intermittent standing bouts improves fatigue and musculoskeletal discomfort in overweight/obese office workers. Occup Environ Med 2014:71:765-71.
31 Wright KP, Bogan RK, Wyatt JK. Shift work and the assessment and management of shift work disorder (SWD). Sleep Med Rev 2013;17:41-54.

32 ABo S. Characteristics of employment. Canberra, ACT: ABS, 2015.

33 Kecklund G, Axelsson J. Health consequences of shift work and insufficient sleep. BMJ 2016;355:i5210.

34 Kivimäki M, Batty GD, Hublin C. Shift work as a risk factor for future type 2 diabetes: evidence, mechanisms, implications, and future research directions. PLoS Med 2011;8:12.

35 Hulsegge G, Gupta N, Holtermann A, et al. Shift workers have similar leisure-time physical activity levels as day workers but are more sedentary at work. Scand J Work Environ Health 2017;43:127-35.

36 Tucker P, Folkard S, Macdonald I. Rest breaks and accident risk. The Lancet 2003;361:680.

37 Tucker P. The impact of rest breaks upon accident risk, fatigue and performance: a review. Work Stress 2003:17:123-37.

38 Sprajcer M, Jay SM, Vincent GE, et al. Uncertain call likelihood negatively affects sleep and next-day cognitive performance while on-call in a laboratory environment. Chronobiol Int 2018;35:838-48.

39 Sargent C, Darwent D, Ferguson SA, et al. Sleep restriction masks the influence of the circadian process on sleep propensity. Chronobiol Int 2012;29:565-71.

40 Zhou X, Ferguson SA, Matthews RW, et al. Sleep, wake and phase dependent changes in neurobehavioral function under forced desynchrony. Sleep 2011;34:931.

41 Gander P, Signal L, Van Dongen HPA, et al. Stable inter-individual differences in slow-wave sleep during nocturnal sleep and naps. Sleep Biol Rhythms 2010;8:239-44.

42 Signal TL, van den Berg MJ, Mulrine HM, et al. Duration of sleep inertia after napping during simulated night work and in extended operations. Chronobiol Int 2012;29:769-79.

43 Buysse DJ, Reynolds CF, Monk TH, et al. The Pittsburgh sleep quality index: a new instrument for psychiatric practice and research. Psychiatry Res 1989;28:193-213.

44 Buysse DJ, Hall ML, Strollo PJ, et al. Relationships between the Pittsburgh sleep quality index (PSQI), Epworth Sleepiness scale (ESS), and clinical/polysomnographic measures in a community sample. J Clin Sleep Med 2008;4:563-71.

45 Backhaus J, Junghanns K, Broocks A, et al. Test-Retest reliability and validity of the Pittsburgh sleep quality index in primary insomnia. J Psychosom Res 2002;53:737-40.

46 Mollayeva T, Thurairajah P, Burton K, et al. The Pittsburgh sleep quality index as a screening tool for sleep dysfunction in clinical and non-clinical samples: a systematic review and meta-analysis. Sleep Med Rev 2016;25:52-73.

47 Johns MW. A new method for measuring daytime sleepiness: the Epworth Sleepiness scale. Sleep 1991;14:540-5.

48 Horne JA, Ostberg O. A self-assessment questionnaire to determine morningness-eveningness in human circadian rhythms. Int $J$ Chronobiol 1976;4:97-110.

49 Netzer NC, Stoohs RA, Netzer CM, et al. Using the Berlin questionnaire to identify patients at risk for the sleep apnea syndrome. Ann Intern Med 1999;131:485-91.

50 Bastien C, Vallieres A, Morin CM. Validation of the insomnia severity index as an outcome measure for insomnia research. Sleep Med 2001;2:297-307.

51 Lovibond PF, Lovibond SH. The structure of negative emotional states: comparison of the depression anxiety stress scales (DASS) with the Beck depression and anxiety inventories. Behav Res Ther 1995;33:335-43.

52 Hagströmer M, Oja P, Sjöström M. The International physical activity questionnaire (IPAQ): a study of concurrent and construct validity. Public Health Nutr 2006;9:755-62.

53 Barnes J, Behrens T, Benden M, et al. Letter to the Editor: Standardized Use of the Terms "Sedentary" and "Sedentary Behaviours". Appl Physiol Nutr Me 2012;37:540-2.

54 Chau JY, van der Ploeg HP, Dunn S, et al. A tool for measuring workers' sitting time by domain: the workforce sitting questionnaire. Br J Sports Med 2011;45:1216-22.

55 Stamatakis E, Gale J, Bauman A, et al. Sitting Time, Physical Activity, and Risk of Mortality in Adults. J Am Coll Cardiol 2019;73:2062-72.

56 Khalesi S, Doshi D, Buys N, et al. Validation of a short food frequency questionnaire in Australian adults. Int $J$ Food Sci Nutr 2017;68:349-57.

57 Khalesi S, Sharma S, Irwin C, et al. Dietary patterns, nutrition knowledge and lifestyle: associations with blood pressure in a sample of Australian adults (the food bp study). J Hum Hypertens 2016:30:581-90. 
58 Norton K, Coombes J, Hobson-Powell A, et al. Adult pre-exercise screening system (APSS), 2011.

59 Allen AM, McRae-Clark AL, Carlson S, et al. Determining menstrual phase in human biobehavioral research: a review with recommendations. Exp Clin Psychopharmacol 2016;24:1-11.

60 Vidafar P, Gooley JJ, Burns AC, et al. Increased vulnerability to attentional failure during acute sleep deprivation in women depends on menstrual phase. Sleep 2018;41:zsy098.

61 Sims ST, Heather AK, Myths HAK. Myths and methodologies: reducing scientific design ambiguity in studies comparing sexes and/or menstrual cycle phases. Exp Physiol 2018;103:1309-17.

62 World Health Organisation. Physical status: the use and interpretation of anthropometry. Report of a who expert Committee. World Health Organ Tech Rep Ser 1995;854:1-452.

63 Kolbe-Alexander TL, Gomersall S, Clark B, et al. A hard day's night: time use in shift workers. BMC Public Health 2019;19:452.

64 Peplonska B, Bukowska A, Sobala W. Association of rotating night shift work with $\mathrm{BMI}$ and abdominal obesity among nurses and midwives. PLoS One 2015;10:e0133761-e.

65 Grant CL, Coates AM, Dorrian J, et al. Timing of food intake during simulated night shift impacts glucose metabolism: a controlled study. Chronobiol Int 2017;34:1003-13.

66 Wehrens SMT, Hampton SM, Kerkhofs M, et al. Mood, alertness, and performance in response to sleep deprivation and recovery sleep in experienced shiftworkers versus non-shiftworkers. Chronobiol Int 2012;29:537-48.

67 World Health Organisation. Waist circumference and waist-hip ratio. Geneva, 2008.

68 Ashwell M, Gunn P, Gibson S. Waist-to-height ratio is a better screening tool than waist circumference and BMI for adult cardiometabolic risk factors: systematic review and meta-analysis. Obes Rev 2012;13:275-86.

69 Borg GAV. Psychophysical bases of perceived exertion. Med sci sports exerc 1982;14:377-81.

70 Bailey DP, Broom DR, Chrismas BCR, et al. Breaking up prolonged sitting time with walking does not affect appetite or gut hormone concentrations but does induce an energy deficit and suppresses postprandial glycaemia in sedentary adults. Appl Physiol Nutr Metab 2016;41:324-31. -

71 Bailey DP, Locke CD. Breaking up prolonged sitting with Lightintensity walking improves postprandial glycemia, but breaking up sitting with standing does not. J Sci Med Sport 2015;18:294-8.

72 Dunstan DW, Kingwell BA, Larsen R, et al. Breaking up prolonged sitting reduces postprandial glucose and insulin responses. Diabetes Care 2012;35:976-83.

73 de Souza L, Benedito-Silva AA, Pires MLN, et al. Further validation of actigraphy for sleep studies. Sleep 2003;26:81-5.

74 Colley R, Connor Gorber S, Tremblay MS. Quality control and data reduction procedures for accelerometry-derived measures of physical activity. Health Rep 2010;21:63.

75 Ridgers ND, Fairclough S. Assessing free-living physical activity using accelerometry: practical issues for researchers and practitioners. Eur J Sport Sci 2011;11:205-13.

76 Welk GJ, Schaben JA, Morrow JR. Reliability of accelerometrybased activity monitors: a generalizability study. Med Sci Sports Exerc 2004;36:1637-45.

77 Chappel S, Aisbett B, Vincent G, et al. Firefighters' physical activity across multiple shifts of planned burn work. Int J Environ Res Public Health 2016;13:973.

78 Aminian S, Hinckson EA. Examining the validity of the ActivPAL monitor in measuring posture and ambulatory movement in children. Int J Behav Nutr Phys Act 2012;9:119.

79 Stanton R, Guertler D, Duncan MJ, et al. Validation of a pouchmounted activPAL3 accelerometer. Gait Posture 2014;40:688-93.

80 Steeves JA, Bowles HR, McClain JJ, et al. Ability of thigh-worn ActiGraph and activPAL monitors to classify posture and motion. Med Sci Sports Exerc 2015;47:952-9.

81 Bassett DR, John D, Conger SA, et al. Detection of lying down, sitting, standing, and stepping using two activPAL monitors. Med Sci Sports Exerc 2014:46:2025-9.

82 Dowd KP, Harrington DM, Bourke AK, et al. The measurement of sedentary patterns and behaviors using the activPAL ${ }^{\mathrm{TM}}$ professional physical activity monitor. Physiol Meas 2012;33:1887-99.

83 An H-S, Kim Y, Lee J-M. Accuracy of inclinometer functions of the activPAL and ActiGraph GT3X+: a focus on physical activity. Gait Posture 2017;51:174-80

84 Rångtell $\mathrm{FH}$, Ekstrand $\mathrm{E}$, Rapp L, et al. Two hours of evening reading on a self-luminous tablet vs. reading a physical book does not alter sleep after daytime bright light exposure. Sleep Med 2016;23:111-8.
85 Zeitzer JM, Dijk D-J, Kronauer RE, et al. Sensitivity of the human circadian pacemaker to nocturnal light: melatonin phase resetting and suppression. J Physiol 2000;526:695-702.

86 Peddie MC, Bone JL, Rehrer NJ, et al. Breaking prolonged sitting reduces postprandial glycemia in healthy, normal-weight adults: a randomized crossover trial. Am J Clin Nutr 2013;98:358-66.

87 Larsen RN, Kingwell BA, Robinson C, et al. Breaking up of prolonged sitting over three days sustains, but does not enhance, lowering of postprandial plasma glucose and insulin in overweight and obese adults. Clin Sci 2015;129:117-27.

88 Dempsey PC, Larsen RN, Sethi P, et al. Benefits for type 2 diabetes of interrupting prolonged sitting with brief bouts of light walking or simple resistance activities. Diabetes Care 2016;39:964-72.

89 Roza AM, Shizgal HM. The Harris Benedict equation reevaluated: resting energy requirements and the body cell mass. Am J Clin Nutr 1984;40:168-82.

90 Gupta CC, Centofanti S, Dorrian J, et al. Altering meal timing to improve cognitive performance during simulated nightshifts. Chronobiol Int 2019;36:1691-713.

91 Gupta CC, Dorrian J, Grant CL, et al. It's not just what you eat but when: the impact of eating a meal during simulated shift work on driving performance. Chronobiol Int 2017;34:66-77.

92 Jung CM, Melanson EL, Frydendall EJ, et al. Energy expenditure during sleep, sleep deprivation and sleep following sleep deprivation in adult humans. J Physiol 2011;589:235-44.

93 Reynolds AC, Dorrian J, Liu PY, et al. Impact of five nights of sleep restriction on glucose metabolism, leptin and testosterone in young adult men. PLoS One 2012;7:e41218.

94 Keenan DB, Mastrototaro JJ, Voskanyan G, et al. Delays in minimally invasive continuous glucose monitoring devices: a review of current technology. J Diabetes Sci Technol 2009;3:1207-14.

95 Larsen RN, Kingwell BA, Sethi P, et al. Breaking up prolonged sitting reduces resting blood pressure in overweight/obese adults. Nutr Metab Cardiovasc Dis 2014;24:976-82.

96 O'Brien E, Coats A, Owens P, et al. Use and interpretation of ambulatory blood pressure monitoring: recommendations of the British hypertension Society. BMJ 2000;320:1128-34.

97 Strath SJ, Swartz AM, Bassett DR, et al. Evaluation of heart rate as a method for assessing moderate intensity physical activity. Med Sci Sports Exerc 2000;32:S465-70.

98 Åkerstedt T, Gillberg M. Subjective and objective sleepiness in the active individual. Int J Neurosci 1990;52:29-37.

99 Samn SW, Perelli LP. Estimating aircrew fatigue: a technique with application to airlift operations, 1982.

100 Watson D, Clark LA, Carey G. Positive and negative affectivity and their relation to anxiety and depressive disorders. J Abnorm Psychol 1988:97:346-53.

101 Jay SM, Lamond N, Ferguson SA, et al. The characteristics of recovery sleep when recovery opportunity is restricted. Sleep 2007;30:353-60.

102 Dinges DF, Powell JW. Microcomputer analyses of performance on a portable, simple visual RT task during sustained operations. Behavior Res Methods 1985;17:652-5.

103 Killgore WD. Effects of sleep deprivation on cognition. In: Kerkhof $\mathrm{G}$, Van Dongen H, eds. Progress in brain research. Amsterdam, Netherlands: Elsevier Science, 2010: 105-30.

104 Dinges DF, Pack F, Williams K, et al. Cumulative sleepiness, mood disturbance, and psychomotor vigilance performance decrements during a week of sleep restricted to $4-5$ hours per night. Sleep 1997:20:267-77.

105 Dinges D, Rogers NL, Dorrian J. Psychomotor vigilance performance: Neurocognitive assay sensitive to sleep loss. In: Kushida C, ed. Sleep deprivation: clinical issues, pharmacology, and sleep loss effects. New York: CRC Press, 2004: 67-98.

106 Van Dongen HPA, Maislin G, Mullington JM, et al. The cumulative cost of additional wakefulness: dose-response effects on neurobehavioral functions and sleep physiology from chronic sleep restriction and total sleep deprivation. Sleep 2003;26:117-26.

107 Lim J, Dinges DF. Sleep deprivation and vigilant attention. Ann N Y Acad Sci 2008;1129:305-22.

108 Jewett ME, Dijk DJ, Kronauer RE, et al. Dose-Response relationship between sleep duration and human psychomotor vigilance and subjective alertness. Sleep 1999;22:171-9.

109 Wechsler D. The measurement and appraisal of adult intelligence, 1958.

110 Stroop JR. Studies of interference in serial verbal reactions. J Exp Psychol 1935;18:643-62.

111 Scarpina F, Tagini S, SJFip T. The Stroop color and word test. Front Psychol 2017;8:557. 
112 Lingenfelser T, Kaschel R, Weber A, et al. Young Hospital doctors after night duty: their task-specific cognitive status and emotional condition. Med Educ 1994;28:566-72.

113 Stenuit P, Kerkhofs M. Effects of sleep restriction on cognition in women. Biol Psychol 2008;77:81-8.

114 Chang Y-K, Pan C-Y, Chen F-T, et al. Effect of resistance-exercise training on cognitive function in healthy older adults: a review. $J$ Aging Phys Act 2012;20:497-517.

115 Cain SW, Silva EJ, Chang A-M, et al. One night of sleep deprivation affects reaction time, but not interference or facilitation in a Stroop task. Brain Cogn 2011;76:37-42.

116 Iber C. The AASM manual for the scoring of sleep and associated events: rules, terminology and technical specifications. Darian, IL, USA: American Adacemy of Sleep Medicine, 2007.

117 Kaida K, Takahashi M, Åkerstedt T, et al. Validation of the Karolinska sleepiness scale against performance and EEG variables. Clin Neurophysiol 2006;117:1574-81.

118 Thayer RE. Activation-Deactivation adjective check list: current overview and structural analysis. Psychol Rep 1986;58:607-14.

119 Galy E, Paxion J, Berthelon C. Measuring mental workload with the NASA-TLX needs to examine each dimension rather than relying on the global score: an example with driving. Ergonomics 2018;61:517-27.

120 Bulmash EL, Moller HJ, Kayumov L, et al. Psychomotor disturbance in depression: assessment using a driving simulator paradigm. $J$ Affect Disord 2006;93:213-8.

121 Blane A, Lee H, Falkmer T, et al. Cognitive ability as a predictor of task demand and self-rated driving performance in poststroke drivers - implications for self-regulation. J Transp Health 2018;9:169-79.

122 Pauzié A. A method to assess the driver mental workload: the driving activity load index (DALI). IET Intelligent Transport Systems 2008;2:315-22.

123 Larue GS, Rakotonirainy A, Haworth NL. A simulator evaluation of effects of assistive technologies on driver cognitive load at railwaylevel crossings. J Transp Saf Secur 2016;8:sup1:56-69.
124 Dorrian J, Baulk SD, Dawson D. Work hours, workload, sleep and fatigue in Australian rail industry employees. Appl Ergon 2011;42:202-9.

125 Perrin SL, Jay SM, Vincent GE, et al. Waking qEEG to assess psychophysiological stress and alertness during simulated on-call conditions. Int J Psychophysiol 2019;141:93-100.

126 Australian Institute of Health and Welfare. The active Australia survey: a guide and manual for implementation analysis and reporting. Canberra, 2003.

127 Armstrong T, Bauman AE, Davies J. Physical activity patterns of Australian adults: results of the 1999. National Physical Activity Survey: Australian Institute of Health and Welfare, 2000.

128 Thorpe MG, Milte CM, Crawford D, et al. A revised Australian dietary guideline index and its association with key sociodemographic factors, health behaviors and body mass index in peri-retirement aged adults. Nutrients 2016;8:160.

129 Eysenck H, Eysenck S. Manual for the Eysenck personality inventory. San Diego: Educational and Industrial Testing Service, 1968.

130 Howarth E. A psychometric investigation of Eysenck's personality inventory. J Pers Assess 1976;40:173-85.

131 Bodling AM, Martin T. Eysenck Personality Inventory. In: Kreutzer JS, DeLuca J, Caplan B, eds. Encyclopedia of clinical neuropsychology. New York, NY: Springer New York, 2011: 1007-8.

132 Rotter JB. Generalized expectancies for internal versus external control of reinforcement. Psychol Monogr 1966;80:1-28.

133 Roenneberg T, Wirz-Justice A, Merrow M. Life between clocks: daily temporal patterns of human chronotypes. J Biol Rhythms 2003:18:80-90.

134 Zavada A, Gordijn MCM, Beersma DGM, et al. Comparison of the Munich Chronotype questionnaire with the Horne-Ostberg's Morningness-Eveningness score. Chronobiol Int 2005;22:267-78

135 Spielberger CD. Manual for the State-Trait Anxiety Inventory STAI (form Y)(" self-evaluation questionnaire". Palo Alto, CA: Consulting Psychologists Press, 1983.

136 IPAQ Research Committee. Guidelines for data processing and analysis of the International Physical Activity Questionnaire (IPAQ)short and long forms, 2005. 